\title{
Free energy landscapes from SARS-CoV-2 spike glycoprotein simulations suggest that RBD opening can be modulated via interactions in an allosteric pocket
}

Lucy Fallon ${ }^{1,2 \#}$, Kellon A.A. Belfon ${ }^{1,2 \#}$, Lauren Raguette ${ }^{1,2}$, Yuzhang Wang ${ }^{1,2}$, Darya Stepanenko ${ }^{1,4}$, Abbigayle Cuomo $^{2}$, Jose Guerra ${ }^{5}$, Stephanie Budhan ${ }^{2}$, Sarah Varghese ${ }^{6}$, Christopher Corbo ${ }^{3}$, Robert C. Rizzo ${ }^{1,4}$, and Carlos Simmerling ${ }^{1,2 *}$

${ }^{1}$ Laufer Center for Physical and Quantitative Biology, Stony Brook University, Stony Brook, New York 11794, United States

${ }^{2}$ Department of Chemistry, Stony Brook University, Stony Brook, New York 11794, United States

${ }^{3}$ Graduate Program in Molecular and Cellular Pharmacology, Stony Brook University, Stony Brook, New York 11794, United States

${ }^{4}$ Department of Applied Mathematics and Statistics, Stony Brook University, Stony Brook, New York 11794, United States

${ }^{5}$ Department of Biochemistry and Cell Biology, Stony Brook University, Stony Brook, New York 11794, United States

${ }^{6}$ Undergraduate Program in Biology, Stony Brook University, Stony Brook, New York 11794, United States

\# These authors contributed equally to this work.

\section{Organization}

1. Figures

2. Tables

3. References

1. Figures
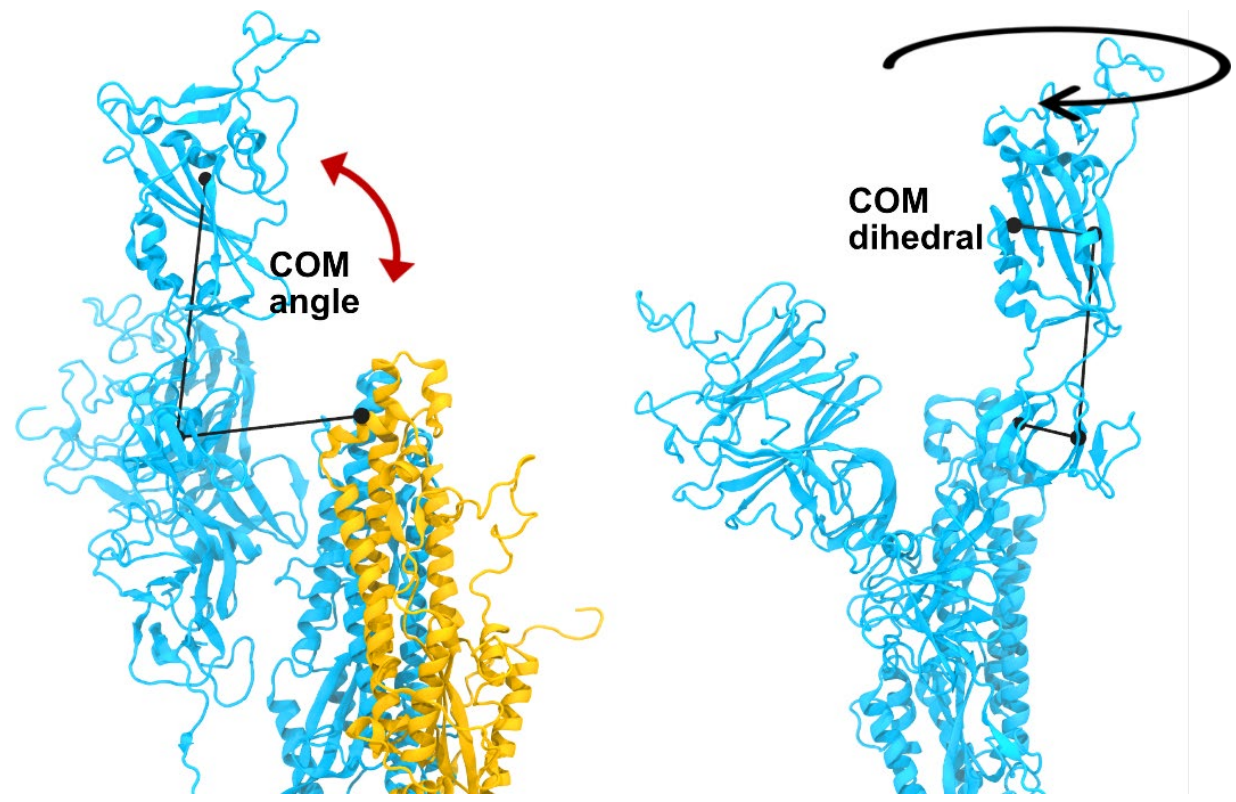
Figure S1- Center of mass (COM) based collective variables (CVs) used to quantify RBD motion relative to the remainder of the spike. (Left) the COM angle measures movement of the RBD up and away from the core central helices, and (right) the COM dihedral measures rotation of the RBD relative to the CTD1 domain. Portions of 2 protomers are shown, with coloring matching Figure 4. Amino acid ranges used to define the COM groups (black circles) are shown in Table S4. Additional details are provided in Methods.
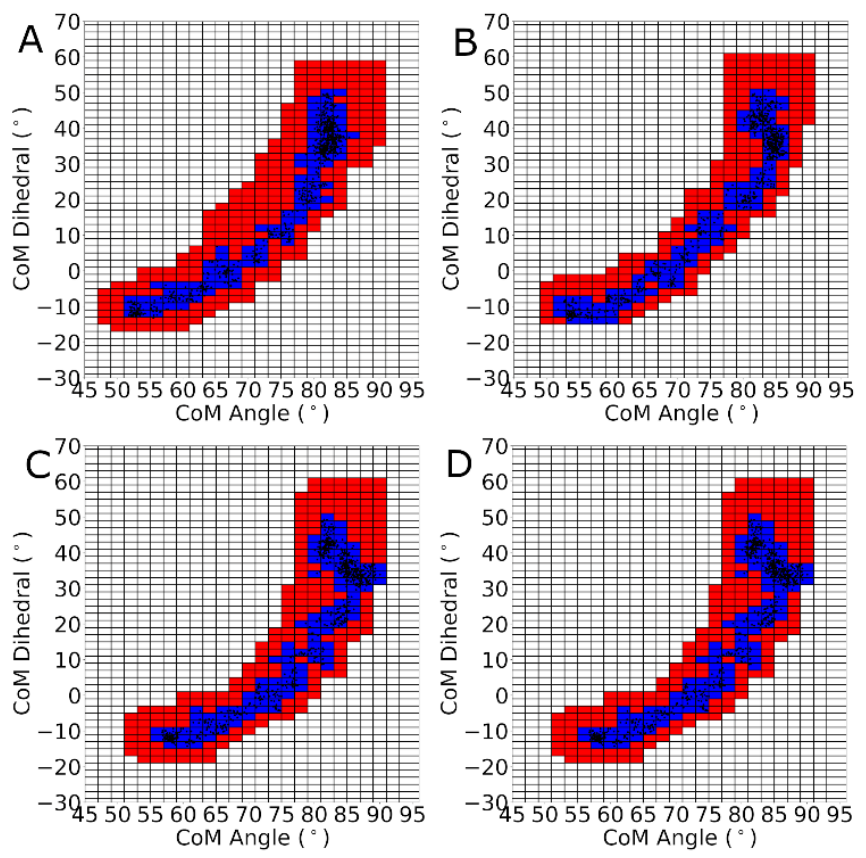

Figure S2: 2D grid of CoM angle and CoM dihedral collective variables. The colored grids were used as windows for US simulations to calculate the free energy for RBD opening in A) WT, B) K528A, C) A522L, and D) A522V Sprotein. The initial grids used as US windows before grid expansion are shown in blue, additional grids used as new windows for expansion are shown in red. The SMD trajectory snapshots are mapped onto the 2D grid as black dots. 

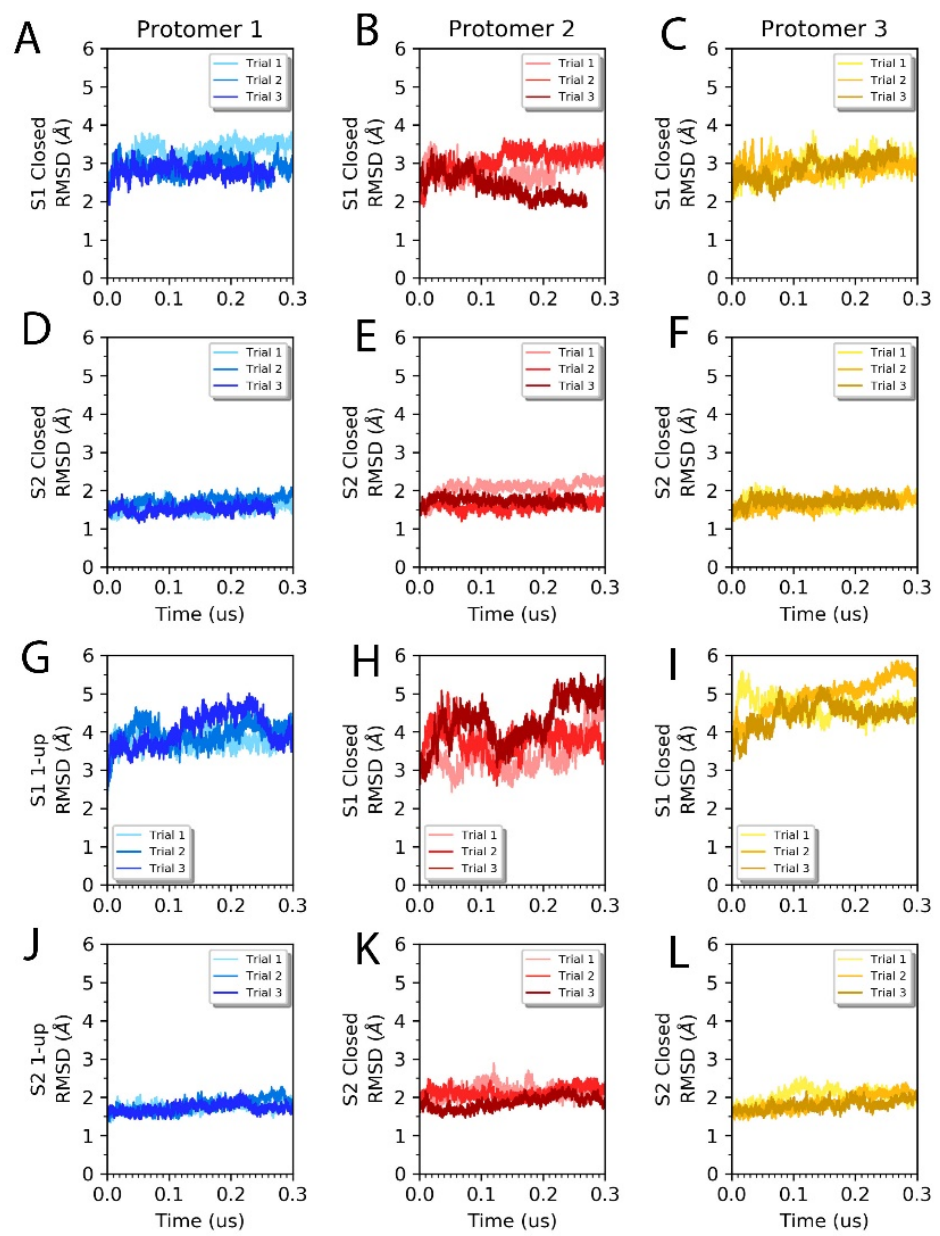

Figure S3. RMSD of backbone atoms of each protomer (blue, red, yellow) over three MD runs of the spike over 270, 300 and 300 ns. (A,B,C) S1, closed spike system; (D,E,F) S2, closed; (G,H,I) S1, 1-up; (J,K,L) S2, 1-up. Each shade in a plot represents one of three independent trials. Columns represent protomers 1,2 and 3 with colors matching Figure 4. RMSD is relative to the respective reference structure. 

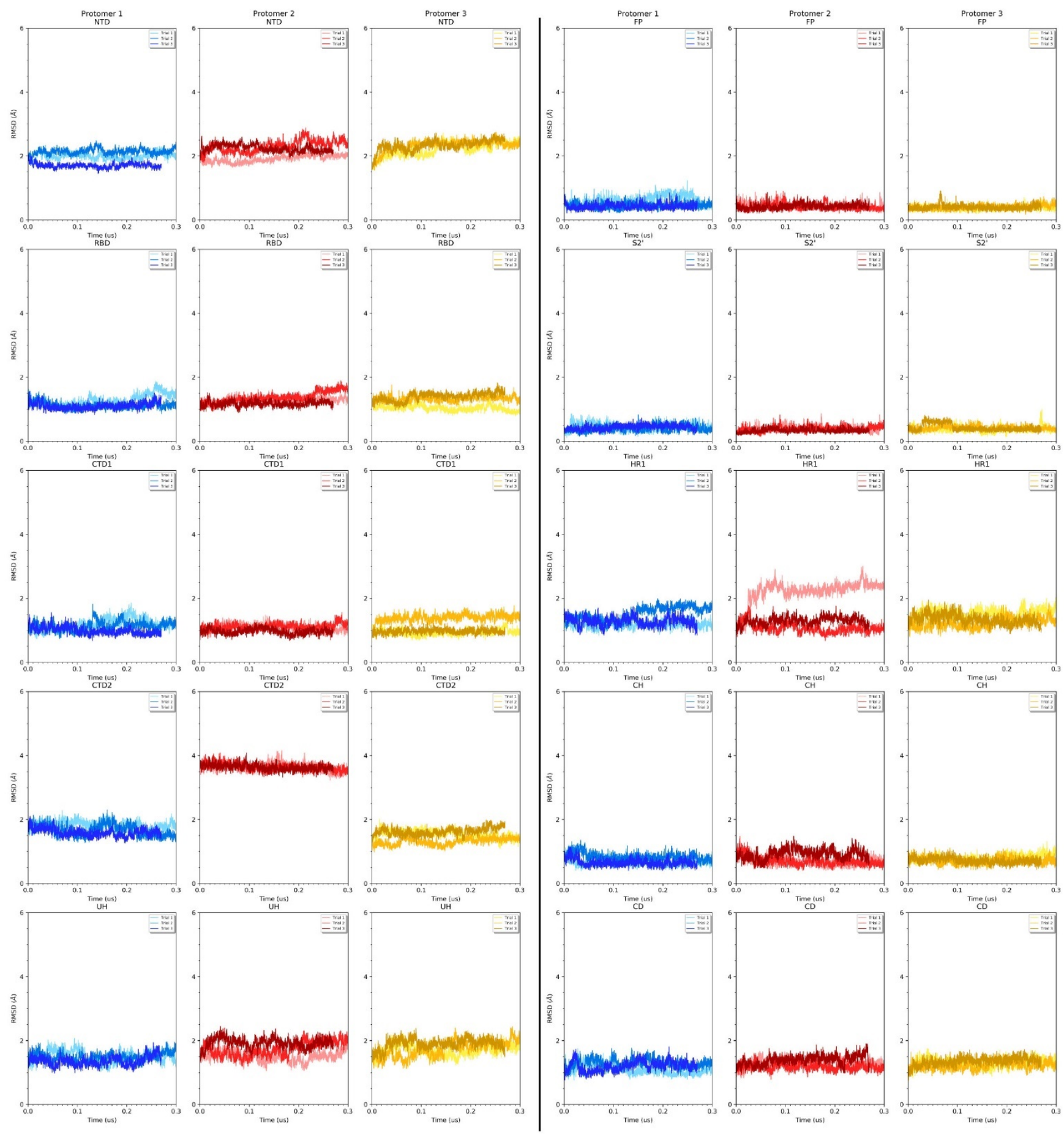

Figure S4. Domain RMSDS during MD simulations of the closed spike. Residue ranges for each domain are provided in Table S3. Rows correspond to specific domains. Columns show data for each protomer, with 3 independent runs in different shades. RMSD is relative to the respective reference structure. 

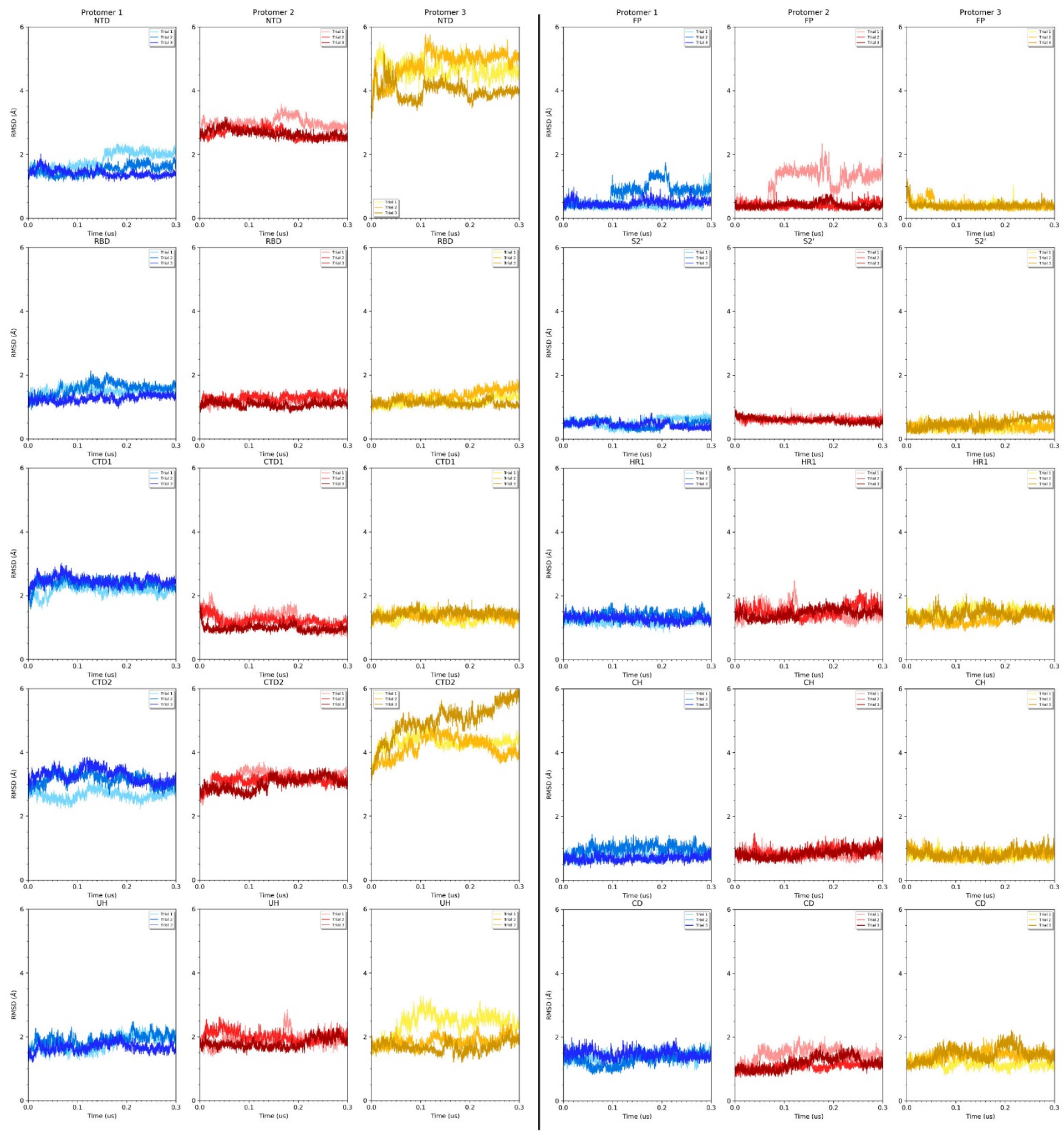

Figure S5. Domain RMSDS during MD simulations of the 1-up spike. Residue ranges for each domain are provided in Table S3. Columns show data for each protomer, with 3 independent runs in different shades. Protomer 1 has the RBD in the open position. RMSD is relative to the respective reference structure. 

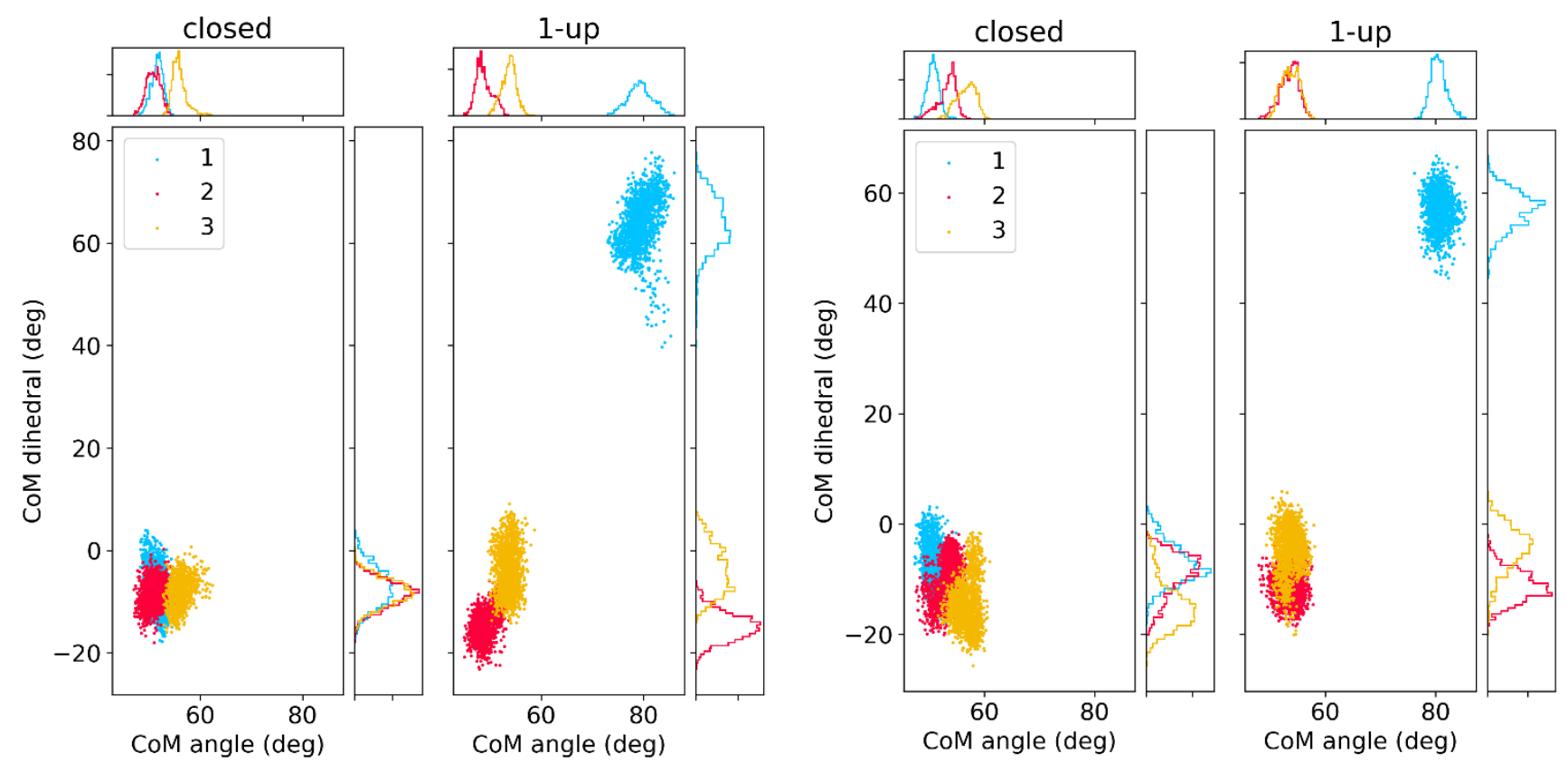

Figure S6. Collective variables for 2 additional independent runs of closed and 1-up spike. Simulation times: 300 ns (left), $270 \mathrm{~ns}$ (right).
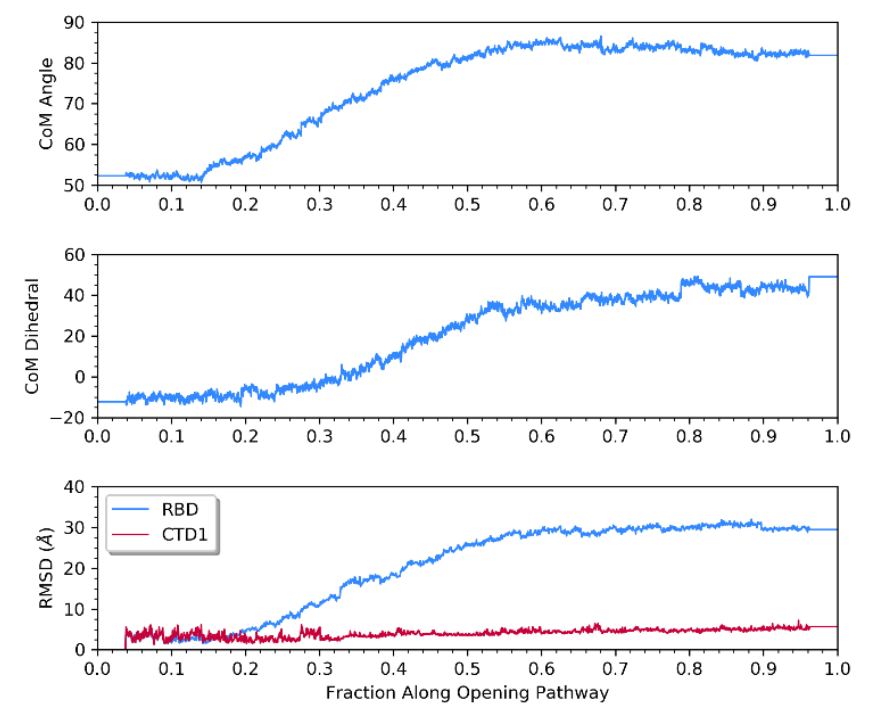

Figure S7. Spike structure properties during the RBD opening pathway, with the closed state on the left and the 1-up spike on the right. (Top) RBD opening angle, (middle) RBD rotation dihedral, (bottom) RMSD values for RBD and CTD1 domains, after best-fit to central helices $(\mathrm{CH})$. 


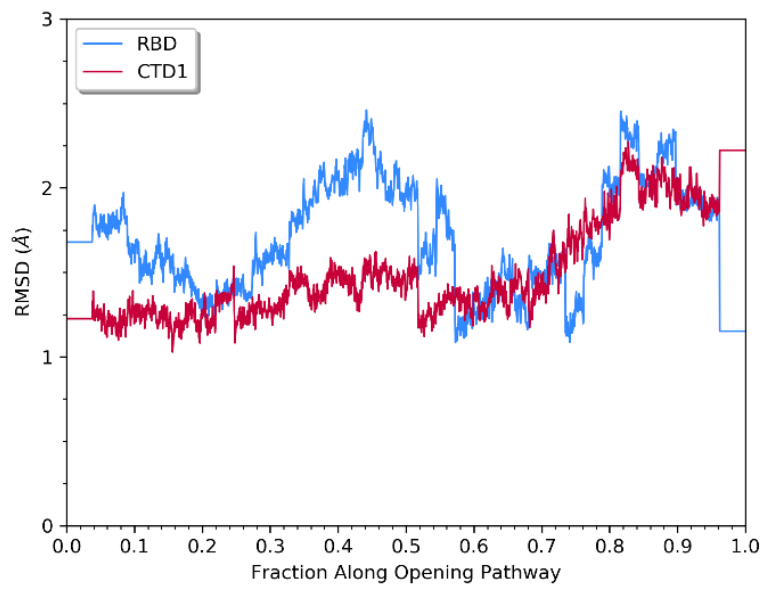

Figure S8. RMSD of RBD and CTD1 domains as a function of opening pathway, with each domain best-fit to the same atoms in the open reference model, using the residues listed in Table S3.
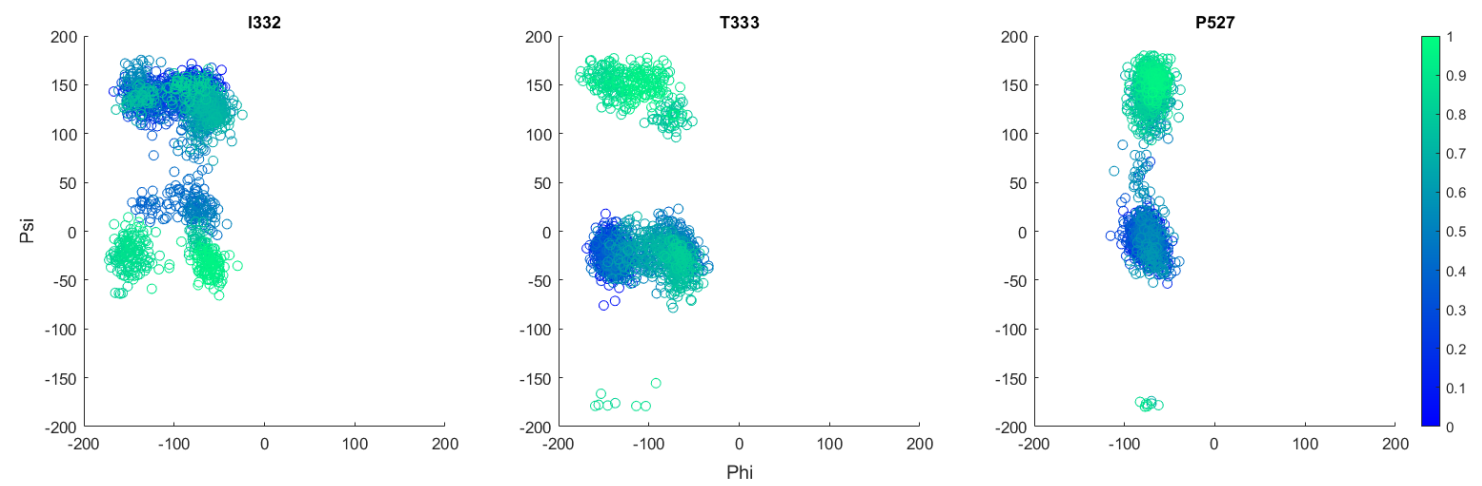

Figure S9. Selected backbone dihedrals in the RBD-CTD1 connector strands as a function of RBD opening pathway. Snapshots are shown as circles, with the opening progress indicated by the color range.

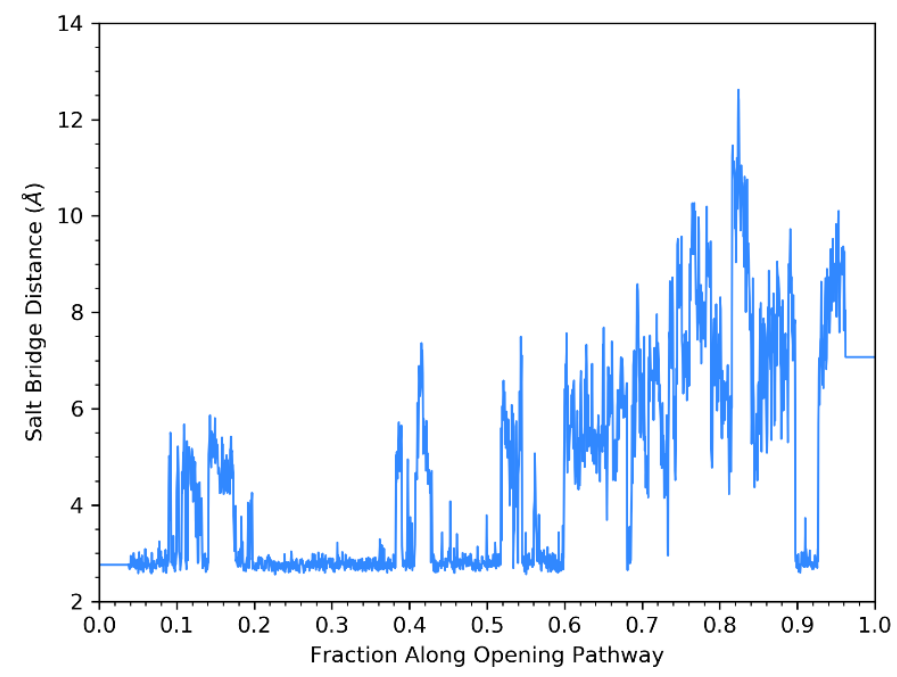

Figure S10. Distance between sidechains of K528 (Nל) and D389 (C $\delta)$ as a function of RBD opening pathway. 


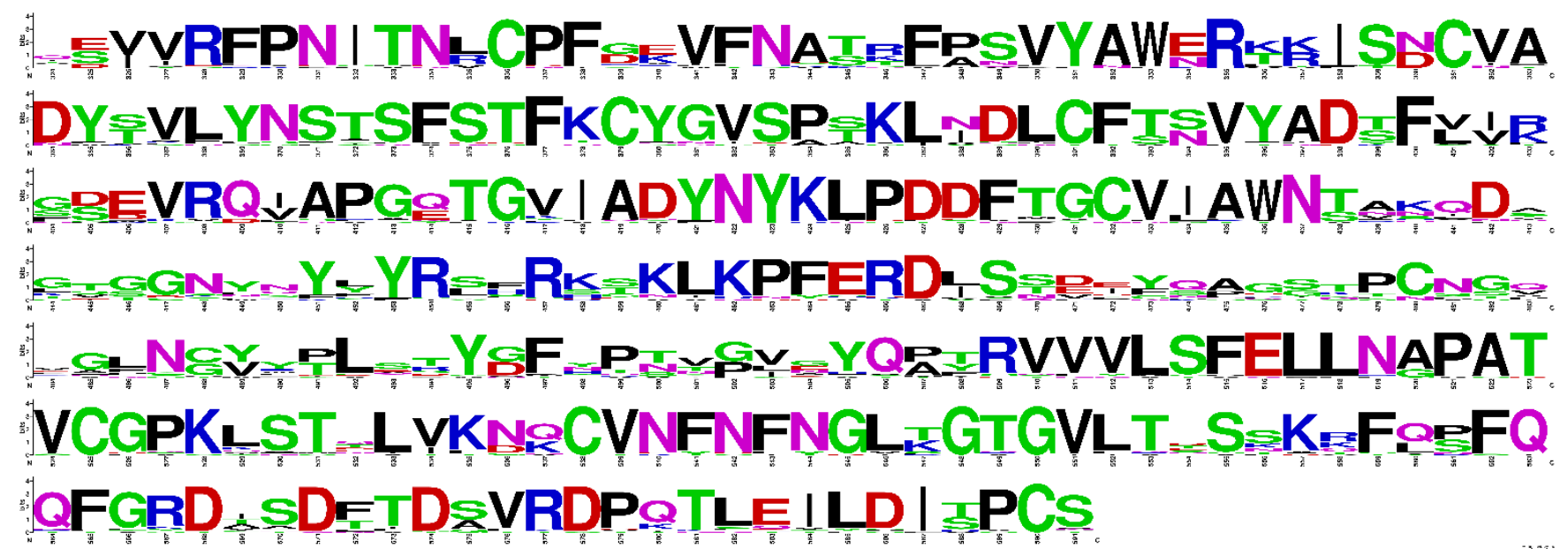

Figure S11. Sequence conservation for amino acids in the hinge pocket region (including positions 324-591), across 21 different coronaviruses.

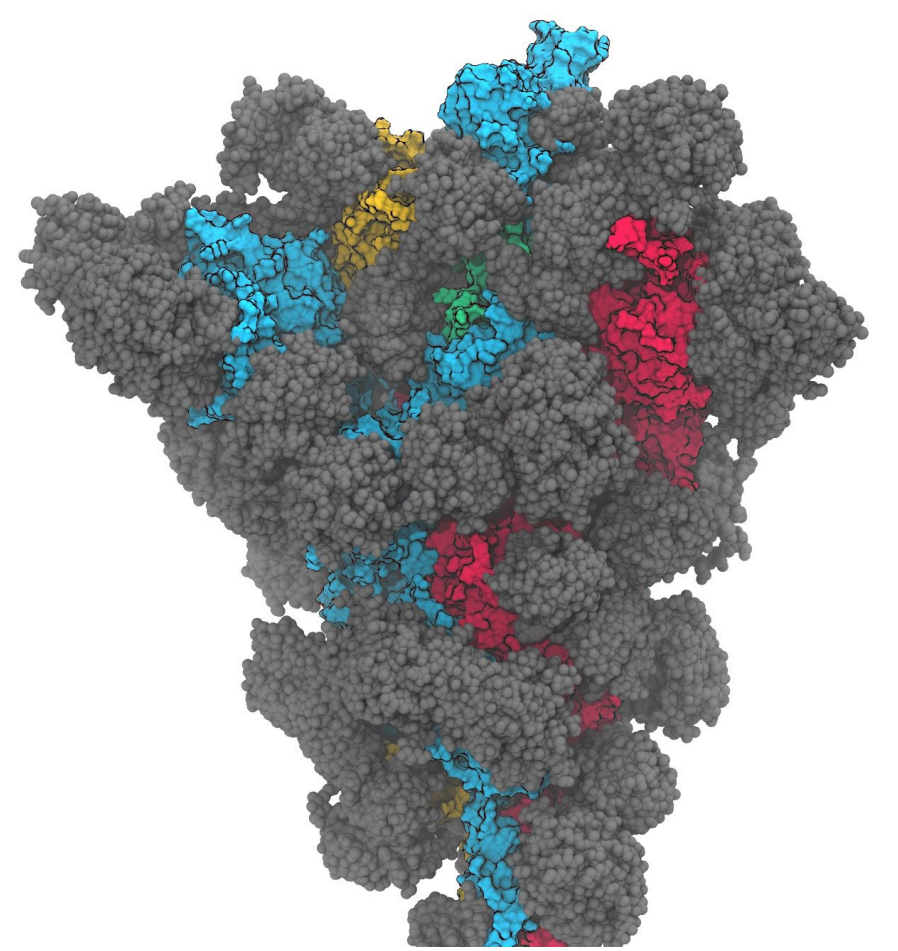

Figure S12. Spike structure in MD simulations, comparable to Figure 3. The gray glycans effectively shield the exterior of the conserved hinge region (highlighted in green). 


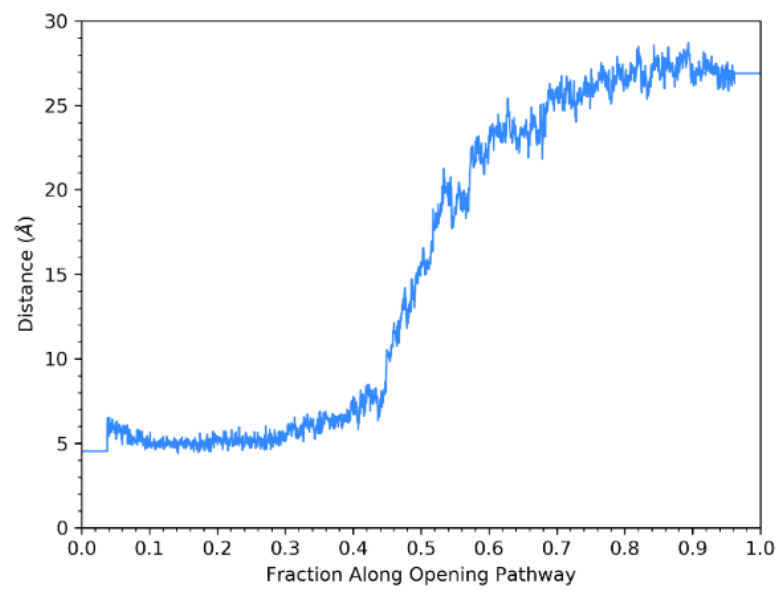

Figure S13. Distance between C $\alpha$ atoms of A520 in RBD and Q564 in CTD1 as a function of RBD opening pathway for full spike;
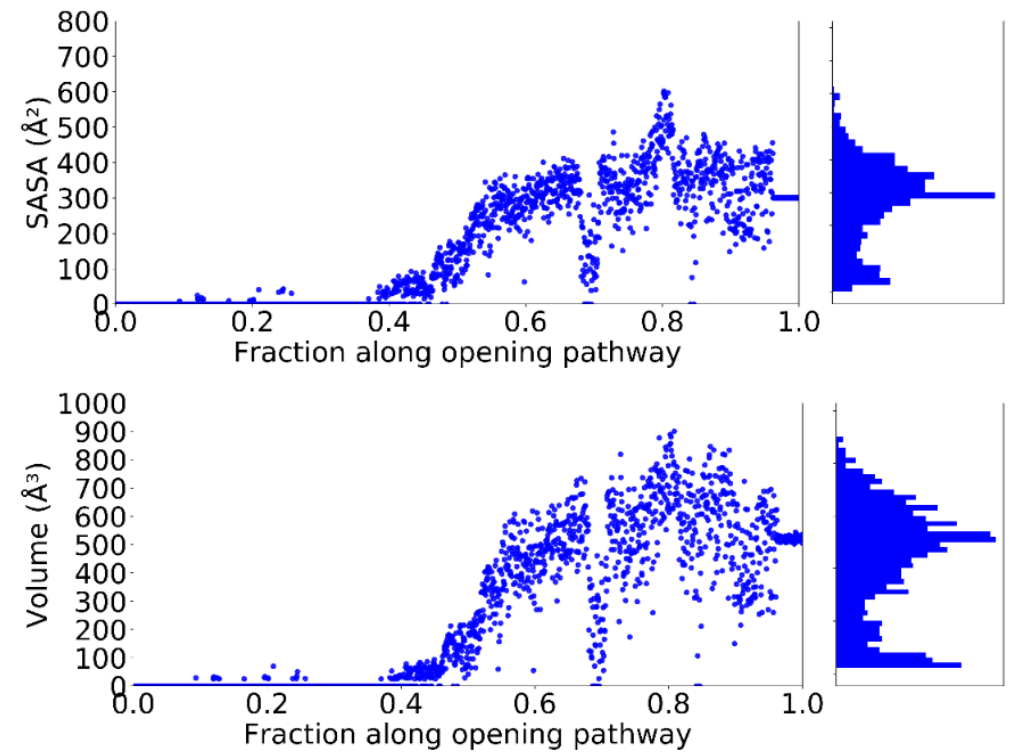

Figure S14: The change in hinge pocket volume and SASA as a function of RBD opening pathway. Data were calculated from the NEB snapshots using fpocket. ${ }^{1}$ 


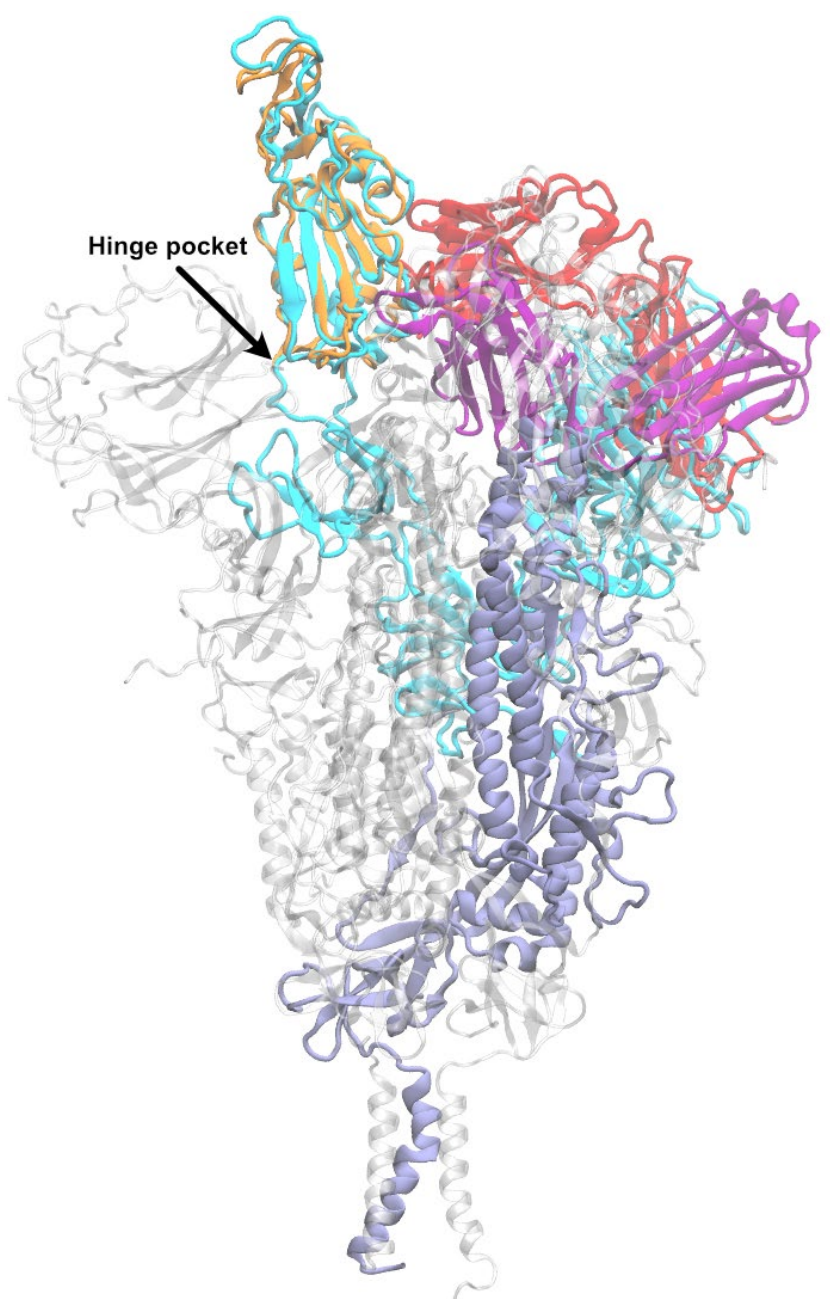

Figure S15. Spike protein from 1-up MD (1 protomer shown with S1 in cyan and S2 in gray, other protomers transparent), with hinge pocket indicated in relation to location of binding of cryptic antibody CR3022 from crystal structure $6 \mathrm{~W} 41^{2}$ (RBD in orange, antibody in red/purple). The crystal structure contained only the RBD, not the entire spike. The RBD domains of the 2 systems were best-fit. 


\section{Tables}

Table S1. Structures and types of glycans on each chain. ${ }^{3}$

\begin{tabular}{|c|c|c|c|c|}
\hline Tyре & Chain A & Chain B & Chain C & Sequence \\
\hline M5 & $\begin{array}{l}\text { N61, N122, } \\
\text { N657 }\end{array}$ & $\begin{array}{l}\text { N61, } \\
\text { N165, } \\
\text { N603, } \\
\text { N709, } \\
\text { N717, } \\
\text { N1074 }\end{array}$ & $\begin{array}{l}\text { N61, } \\
\text { N122, } \\
\text { N603 }\end{array}$ & $\begin{array}{l}\operatorname{aDMan}(1 \rightarrow 6)[\operatorname{aDMan}(1 \rightarrow 3)] \operatorname{aDMan}(1 \rightarrow 6) \\
{[\operatorname{aDMan}(1 \rightarrow 3)] \mathrm{bDMan}(1 \rightarrow 4) \operatorname{bDGIcNAc}(1 \rightarrow 4) \operatorname{bDGIcNAc}(1 \rightarrow)}\end{array}$ \\
\hline M6 & $\begin{array}{l}\text { N709, } \\
\text { N801 }\end{array}$ & & N717 & $\begin{array}{l}\operatorname{aDMan}(1 \rightarrow 6)[\operatorname{aDMan}(1 \rightarrow 3)] \mathrm{aDMan}(1 \rightarrow 6) \\
{[\operatorname{aDMan}(1 \rightarrow 2) \operatorname{aDMan}(1 \rightarrow 3)] \mathrm{bDMan}(1 \rightarrow 4) \mathrm{bDGlcNAc}(1 \rightarrow 4) \mathrm{bDGlcNAc}(} \\
1 \rightarrow)\end{array}$ \\
\hline M7 & & N801 & & $\begin{array}{l}\operatorname{aDMan}(1 \rightarrow 2) \operatorname{aDMan}(1 \rightarrow 2) \operatorname{aDMan}(1 \rightarrow 3)[\operatorname{aDMan}(1 \rightarrow 6) \\
[\operatorname{aDMan}(1 \rightarrow 3)] \mathrm{aDMan}(1 \rightarrow 6)] \mathrm{bDMan}(1 \rightarrow 4) \mathrm{bDGIcNAc}(1 \rightarrow 4) \mathrm{bDGlcNAc}( \\
1 \rightarrow)\end{array}$ \\
\hline M8 & N234 & & & $\begin{array}{l}\operatorname{aDMan}(1 \rightarrow 2) \operatorname{aDMan}(1 \rightarrow 6)[\operatorname{aDMan}(1 \rightarrow 3)] \mathrm{aDMan}(1 \rightarrow 6) \\
{[\operatorname{aDMan}(1 \rightarrow 2) \operatorname{aDMan}(1 \rightarrow 2) \operatorname{aDMan}(1 \rightarrow 3)] \mathrm{bDMan}(1 \rightarrow 4) \mathrm{bDGlcNAc}(1 \rightarrow} \\
\text { 4)bDGIcNAc}(1 \rightarrow)\end{array}$ \\
\hline M9 & & N234 & N234 & $\begin{array}{l}\operatorname{aDMan}(1 \rightarrow 2) \operatorname{aDMan}(1 \rightarrow 6)[\operatorname{aDMan}(1 \rightarrow 2) \mathrm{aDMan}(1 \rightarrow 3)] \mathrm{aDMan}(1 \rightarrow 6) \\
{[\operatorname{aDMan}(1 \rightarrow 2) \operatorname{aDMan}(1 \rightarrow 2) \operatorname{aDMan}(1 \rightarrow 3)] \mathrm{bDMan}(1 \rightarrow 4) \operatorname{bDGIcNAc}(1 \rightarrow} \\
\text { 4)bDGIcNAc}(1 \rightarrow)\end{array}$ \\
\hline A2 & $\begin{array}{l}\text { N616, } \\
\text { N1158 }\end{array}$ & N1098 & $\begin{array}{l}\text { N74, } \\
\text { N282, } \\
\text { N1158 }\end{array}$ & $\begin{array}{l}\text { bDGIcNAc }(1 \rightarrow 2) \mathrm{aDMan}(1 \rightarrow 6) \\
{[\mathrm{bDGlcNAc}(1 \rightarrow 2) \mathrm{aDMan}(1 \rightarrow 3)] \mathrm{bDMan}(1 \rightarrow 4) \mathrm{bDGlcNAc}(1 \rightarrow 4) \mathrm{bDGlcNA}} \\
\mathrm{c}(1 \rightarrow)\end{array}$ \\
\hline A3 & N74 & & & $\begin{array}{l}\text { bDGIcNAc }(1 \rightarrow 6)[\mathrm{bDGlcNAc}(1 \rightarrow 2)] \mathrm{aDMan}(1 \rightarrow 6) \\
{[\mathrm{bDGlcNAc}(1 \rightarrow 2) \mathrm{aDMan}(1 \rightarrow 3)] \mathrm{bDMan}(1 \rightarrow 4) \mathrm{bDGlcNAc}(1 \rightarrow 4) \mathrm{bDGlcNA}} \\
\mathrm{c}(1 \rightarrow)\end{array}$ \\
\hline FA1 & N1134 & N343 & & $\begin{array}{l}\text { bDGIcNAc }(1 \rightarrow 2) \text { aDMan }(1 \rightarrow 3)[\operatorname{aDMan}(1 \rightarrow 6)] \mathrm{bDMan}(1 \rightarrow 4) \mathrm{bDGIcNAc}( \\
1 \rightarrow 4)[\operatorname{aLFuc}(1 \rightarrow 6)] \mathrm{bDGIcNAc}(1 \rightarrow)\end{array}$ \\
\hline FA2 & $\begin{array}{l}\text { N17, N331, } \\
\text { N343, } \\
\text { N603, } \\
\text { N1098 }\end{array}$ & $\begin{array}{l}\text { N122, } \\
\text { N331, } \\
\text { N616 }\end{array}$ & $\begin{array}{l}\text { N149, } \\
\text { N343, } \\
\text { N616, } \\
\text { N709, } \\
\text { N801, } \\
\text { N1074, } \\
\text { N1134 }\end{array}$ & $\begin{array}{l}\text { bDGIcNAc }(1 \rightarrow 2) \text { aDMan }(1 \rightarrow 6)[\mathrm{bDGlcNAc}(1 \rightarrow 2) \operatorname{aDMan}(1 \rightarrow 3)] \mathrm{bDMan}( \\
1 \rightarrow 4) \mathrm{bDGIcNAc}(1 \rightarrow 4)[\operatorname{aLFuc}(1 \rightarrow 6)] \mathrm{bDGIcNAc}(1 \rightarrow)\end{array}$ \\
\hline FA2G2S1 & $\begin{array}{l}\text { N149, } \\
\text { N1074 }\end{array}$ & N1158 & N165 & $\begin{array}{l}\text { aDNeu5Ac }(2 \rightarrow 6) \mathrm{bDGal}(1 \rightarrow 4) \mathrm{bDGlcNAc}(1 \rightarrow 2) \mathrm{aDMan}(1 \rightarrow 6) \\
{[\mathrm{bDGal}(1 \rightarrow 4) \mathrm{bDGlcNAc}(1 \rightarrow 2) \mathrm{aDMan}(1 \rightarrow 3)] \mathrm{bDMan}(1 \rightarrow 4) \mathrm{bDGIcNAc}(1} \\
\rightarrow 4)[\mathrm{aLFuc}(1 \rightarrow 6)] \mathrm{bDGlcNAc}(1 \rightarrow)\end{array}$ \\
\hline FA2G2S2 & N165 & & & $\begin{array}{l}\text { xaDNeu5Ac }(2 \rightarrow 6) \mathrm{bDGal}(1 \rightarrow 4) \mathrm{bDGlcNAc}(1 \rightarrow 2) \operatorname{aDMan}(1 \rightarrow 6) \\
{[\mathrm{aDNeu} 5 \mathrm{Ac}(2 \rightarrow 6) \mathrm{bDGal}(1 \rightarrow 4) \mathrm{bDGlcNAc}(1 \rightarrow 2) \mathrm{aDMan}(1 \rightarrow 3)] \mathrm{bDMan}(1} \\
\rightarrow 4) \operatorname{bDGIcNAc}(1 \rightarrow 4)[\operatorname{aLFuc}(1 \rightarrow 6)] \mathrm{bDGlcNAc}(1 \rightarrow)\end{array}$ \\
\hline FA3 & N282 & $\begin{array}{l}\text { N17, } \\
\text { N149, } \\
\text { N1134 }\end{array}$ & N17 & $\begin{array}{l}\text { bDGIcNAc }(1 \rightarrow 6)[\mathrm{bDGlcNAc}(1 \rightarrow 2)] \mathrm{aDMan}(1 \rightarrow 6) \\
{[\mathrm{bDGIcNAc}(1 \rightarrow 2) \mathrm{aDMan}(1 \rightarrow 3)] \mathrm{bDMan}(1 \rightarrow 4) \mathrm{bDGIcNAc}(1 \rightarrow 4)[\operatorname{aLFuc}(1} \\
\rightarrow 6)] \mathrm{bDGIcNAc}(1 \rightarrow)\end{array}$ \\
\hline
\end{tabular}




\begin{tabular}{|c|c|c|c|c|}
\hline FA3G3S1 & & N282 & N331 & 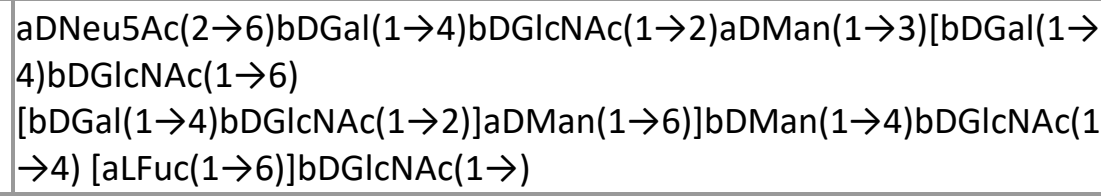 \\
\hline FA3G3S2 & & N74 & & 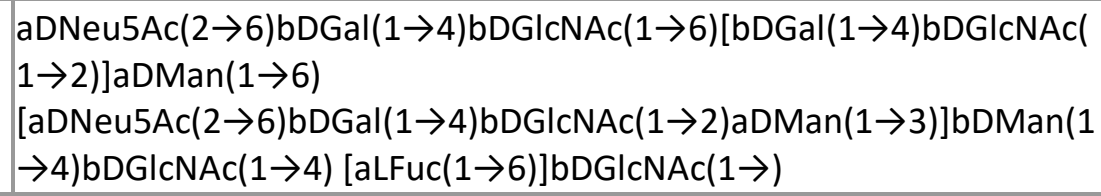 \\
\hline FA4 & N1173 & N1173 & N1173 & $\begin{array}{l}\operatorname{bDGIcNAc}(1 \rightarrow 6)[\mathrm{bDGlcNAc}(1 \rightarrow 2)] \mathrm{aDMan}(1 \rightarrow 6)[\mathrm{bDGlcNAc}(1 \rightarrow 4) \\
[\mathrm{bDGIcNAc}(1 \rightarrow 2)] \mathrm{aDMan}(1 \rightarrow 3)] \mathrm{bDMan}(1 \rightarrow 4) \operatorname{bDGIcNAc}(1 \rightarrow 4)[\operatorname{aLFuc}( \\
1 \rightarrow 6)] \mathrm{bDGIcNAc}(1 \rightarrow)\end{array}$ \\
\hline FA4G4S1 & N1194 & N1194 & N1194 & $\begin{array}{l}\text { aDNeu5Ac }(2 \rightarrow 6) \text { bDGal }(1 \rightarrow 4) \text { bDGlcNAc }(1 \rightarrow 6)[\mathrm{bDGal}(1 \rightarrow 4) \mathrm{bDGlcNAc}( \\
1 \rightarrow 2)] \mathrm{aDMan}(1 \rightarrow 6)[\mathrm{bDGal}(1 \rightarrow 4) \mathrm{bDGlcNAc}(1 \rightarrow 4) \\
[\mathrm{bDGal}(1 \rightarrow 4) \mathrm{bDGlcNAc}(1 \rightarrow 2)] \mathrm{aDMan}(1 \rightarrow 3)] \mathrm{bDMan}(1 \rightarrow 4) \mathrm{bDGlcNAc}(1 \\
\rightarrow 4)[\mathrm{aLFuc}(1 \rightarrow 6)] \mathrm{bDGlcNAc}(1 \rightarrow)\end{array}$ \\
\hline Hybrid G1 & N717 & N657 & N657 & $\begin{array}{l}\operatorname{bDGal}(1 \rightarrow 4) \mathrm{bDGlcNAc}(1 \rightarrow 2) \mathrm{aDMan}(1 \rightarrow 3)[\operatorname{aDMan}(1 \rightarrow 6) \\
[\operatorname{aDMan}(1 \rightarrow 3)] \mathrm{aDMan}(1 \rightarrow 6)] \mathrm{bDMan}(1 \rightarrow 4) \mathrm{bDGIcNAc}(1 \rightarrow 4) \mathrm{bDGlcNAc}( \\
1 \rightarrow)\end{array}$ \\
\hline $\begin{array}{l}\text { Hybrid } \\
\text { G1S1 }\end{array}$ & & & N1098 & $\begin{array}{l}\text { aDNeu5Ac }(2 \rightarrow 6) b D G a l(1 \rightarrow 4) b D G I c N A c(1 \rightarrow 2) \operatorname{aDMan}(1 \rightarrow 3)[\operatorname{aDMan}(1 \\
\rightarrow 6) \\
[\operatorname{aDMan}(1 \rightarrow 3)] \mathrm{aDMan}(1 \rightarrow 6)] \mathrm{bDMan}(1 \rightarrow 4) \mathrm{bDGlcNAc}(1 \rightarrow 4) \mathrm{bDGIcNAc}( \\
1 \rightarrow)\end{array}$ \\
\hline 01 & & & T323 & bDGal $(1 \rightarrow 3)$ aDGalNAc $(1 \rightarrow)$ \\
\hline 02 & T323, S325 & & & aDNeu5Ac $(2 \rightarrow 3)$ bDGal $(1 \rightarrow 3)$ aDGalNAc $(1 \rightarrow)$ \\
\hline 03 & & T323 & & $\operatorname{aDNeu} 5 \mathrm{Ac}(2 \rightarrow 3) \mathrm{bDGal}(1 \rightarrow 3)[\operatorname{aDNeu} 5 \mathrm{Ac}(2 \rightarrow 6)] \mathrm{aDGaINAc}(1 \rightarrow)$ \\
\hline
\end{tabular}

Table S2. Disulfide bonds in the "amended models". Rows in bold indicate the five disulfide bonds in the RBD and CTD1 domains, also included in the RBD+CTD1 construct.

\begin{tabular}{|l|}
\hline SARS-CoV-2 \\
\hline C131 - C166 \\
\hline C291 - C301 \\
\hline C336 - C361 \\
\hline C379 - C432 \\
\hline C391 - C525 \\
\hline C480 - C488 \\
\hline C538 - C590 \\
\hline C617 - C649 \\
\hline C662 - C671 \\
\hline C730 - C768 \\
\hline C743 - C749 \\
\hline C1032 - C1043 \\
\hline C1082 - C1126 \\
\hline
\end{tabular}


Table S3. Reside ranges used for RMSD calculations

\begin{tabular}{|l|l|l|l|}
\hline Subunit & $\begin{array}{l}\text { Domain/ } \\
\text { Region of interest }\end{array}$ & Residues in domain & Residues for RMSD \\
\hline S1 & NTD & $16-285$ & $\begin{array}{l}27-66,81-140,164-172, \\
186-196,200-211,215- \\
242,263-285\end{array}$ \\
\cline { 2 - 4 } & RBD & & $\begin{array}{l}335-454,462-466,491- \\
515,522-530\end{array}$ \\
\cline { 2 - 4 } & CTD1 & $335-530$ & $315-334,531-591$ \\
\hline \multirow{5}{*}{ S2 } & CTD2 & $315-334,531-591$ & $592-620,641-676$ \\
\hline & UH & $592-685$ & $691-811,813$ \\
\hline & S2' (KRSF) & $686-813$ & $814-817$ \\
\hline & FP & $814-817$ & $818-825$ \\
\hline & HR1 & $818-825$ & $867-984$ \\
\cline { 2 - 4 } & CH & $867-984$ & $985-1036$ \\
\cline { 2 - 4 } & CD & $1037-1036$ & $1037-1137$ \\
\hline
\end{tabular}

Table S4. Amino acids used to define center-of-mass collective variables for the RBD closed-open transition. The group is defined by the center of mass of all $\mathrm{C} \alpha$ atoms of all amino acids in the range.

\begin{tabular}{|l|l|l|l|l|}
\hline CoM groups & 1 & 2 & 3 & 4 \\
\hline Angle & F338-L517 & $\begin{array}{l}\text { E324-V327, } \\
\text { C538-L585 }\end{array}$ & T747*-Q755* & \\
\hline Dihedral & $\begin{array}{l}\text { E324-V327, } \\
\text { C538-F543, } \\
\text { L546-T549 }\end{array}$ & $\begin{array}{l}\text { A575-D578, } \\
\text { L582-D586 }\end{array}$ & $\begin{array}{l}\text { N354-I358, } \\
\text { T396-S399 }\end{array}$ & $\begin{array}{l}\text { S375-C379, } \\
\text { C432-W436 }\end{array}$ \\
\hline
\end{tabular}

Asterisks indicates the neighboring protomer on the clockwise side when viewing the spike from the S1 side (top side)

Table S5. List of sequences used to calculate conservation scores. We gratefully acknowledge the following Authors from the Originating laboratories responsible for obtaining the specimens and the Submitting laboratories where genetic sequence data were generated and shared via the GISAID Initiative, on which this research is based. All submitters of data may be contacted directly via www.gisaid.org ${ }^{4}$

\begin{tabular}{|l|l|l|l|l|l|l|l|}
\hline Accession ID & Virus Name & Location & $\begin{array}{l}\text { Date } \\
\text { Collect } \\
\text { ed }\end{array}$ & Host & $\begin{array}{l}\text { Originatin } \\
\text { g Lab }\end{array}$ & $\begin{array}{l}\text { Submittin } \\
\text { g Lab }\end{array}$ & Authors \\
\hline $\begin{array}{l}\text { EPI_ISL_4021 } \\
24\end{array}$ & $\begin{array}{l}\text { hCoV- } \\
19 / \text { Wuhan/WIV04/2019 } \\
\text { (SARS-CoV-2) }\end{array}$ & $\begin{array}{l}\text { Asia / } \\
\text { China / } \\
\text { Hubei / } \\
\text { Wuhan }\end{array}$ & $\begin{array}{l}2019- \\
12-30\end{array}$ & $\begin{array}{l}\text { Homo } \\
\text { sapiens }\end{array}$ & $\begin{array}{l}\text { Wuhan } \\
\text { Jinyintan } \\
\text { Hospital }\end{array}$ & $\begin{array}{l}\text { Wuhan } \\
\text { Institute of } \\
\text { Virology, } \\
\text { Chinese } \\
\text { Academy } \\
\text { of } \\
\text { Sciences }\end{array}$ & $\begin{array}{l}\text { Peng Zhou, Xing-Lou } \\
\text { Lang, Zing, Yan Zhang, } \\
\text { Hao-Rui Si, Zhengli Shi }\end{array}$ \\
\hline
\end{tabular}




\begin{tabular}{|c|c|c|c|c|c|c|c|}
\hline $\begin{array}{l}\text { EPI_ISL_4105 } \\
39\end{array}$ & $\begin{array}{l}\text { hCoV- } \\
\text { 19/pangolin/Guangxi/P1E } \\
\text { /2017 }\end{array}$ & $\begin{array}{l}\text { Asia / } \\
\text { China / } \\
\text { Guangxi }\end{array}$ & 2017 & $\begin{array}{l}\text { Manis } \\
\text { javanica }\end{array}$ & \begin{tabular}{|l} 
Beijing \\
Institute of \\
Microbiolo \\
gy and \\
Epidemiol \\
ogy
\end{tabular} & $\begin{array}{l}\text { Beijing } \\
\text { Institute of } \\
\text { Microbiolo } \\
\text { gy and } \\
\text { Epidemiol } \\
\text { ogy }\end{array}$ & $\begin{array}{l}\text { Wu-Chun Cao; } \\
\text { Tommy Tsan-Yuk } \\
\text { Lam; Na Jia; Ya-Wei } \\
\text { Zhang; Jia-Fu Jiang; } \\
\text { Bao-Gui Jiang }\end{array}$ \\
\hline $\begin{array}{l}\text { EPI_ISL_4105 } \\
41\end{array}$ & $\begin{array}{l}\text { hCoV- } \\
\text { 19/pangolin/Guangxi/P5E } \\
\text { /2017 }\end{array}$ & $\begin{array}{l}\text { Asia / } \\
\text { China / } \\
\text { Guangxi }\end{array}$ & 2017 & $\begin{array}{l}\text { Manis } \\
\text { javanica }\end{array}$ & \begin{tabular}{|l} 
Beijing \\
Institute of \\
Microbiolo \\
gy and \\
Epidemiol \\
ogy
\end{tabular} & $\begin{array}{l}\text { Beijing } \\
\text { Institute of } \\
\text { Microbiolo } \\
\text { gy and } \\
\text { Epidemiol } \\
\text { ogy }\end{array}$ & $\begin{array}{l}\text { Wu-Chun Cao; } \\
\text { Tommy Tsan-Yuk } \\
\text { Lam; Na Jia; Ya-Wei } \\
\text { Zhang; Jia-Fu Jiang; } \\
\text { Bao-Gui Jiang }\end{array}$ \\
\hline $\begin{array}{l}\text { EPI_ISL_4105 } \\
40\end{array}$ & $\begin{array}{l}\text { hCoV- } \\
\text { 19/pangolin/Guangxi/P5L/ } \\
2017\end{array}$ & $\begin{array}{l}\text { Asia / } \\
\text { China / } \\
\text { Guangxi }\end{array}$ & 2017 & $\begin{array}{l}\text { Manis } \\
\text { javanica }\end{array}$ & \begin{tabular}{|l} 
Beijing \\
Institute of \\
Microbiolo \\
gy and \\
Epidemiol \\
ogy
\end{tabular} & $\begin{array}{l}\text { Beijing } \\
\text { Institute of } \\
\text { Microbiolo } \\
\text { gy and } \\
\text { Epidemiol } \\
\text { ogy }\end{array}$ & $\begin{array}{l}\text { Wu-Chun Cao; } \\
\text { Tommy Tsan-Yuk } \\
\text { Lam; Na Jia; Ya-Wei } \\
\text { Zhang; Jia-Fu Jiang; } \\
\text { Bao-Gui Jiang }\end{array}$ \\
\hline $\begin{array}{l}\text { EPI_ISL_4105 } \\
38\end{array}$ & $\begin{array}{l}\text { hCoV- } \\
\text { 19/pangolin/Guangxi/P4L/ } \\
2017\end{array}$ & $\begin{array}{l}\text { Asia / } \\
\text { China / } \\
\text { Guangxi }\end{array}$ & 2017 & $\begin{array}{l}\text { Manis } \\
\text { javanica }\end{array}$ & \begin{tabular}{|l|} 
Beijing \\
Institute of \\
Microbiolo \\
gy and \\
Epidemiol \\
ogy
\end{tabular} & \begin{tabular}{|l} 
Beijing \\
Institute of \\
Microbiolo \\
gy and \\
Epidemiol \\
ogy
\end{tabular} & $\begin{array}{l}\text { Wu-Chun Cao; } \\
\text { Tommy Tsan-Yuk } \\
\text { Lam; Na Jia; Ya-Wei } \\
\text { Zhang; Jia-Fu Jiang; } \\
\text { Bao-Gui Jiang }\end{array}$ \\
\hline $\begin{array}{l}\text { EPI_ISL_4105 } \\
42\end{array}$ & $\begin{array}{l}\text { hCoV- } \\
\text { 19/pangolin/Guangxi/P2V } \\
\text { /2017 }\end{array}$ & $\begin{array}{l}\text { Asia / } \\
\text { China / } \\
\text { Guangxi }\end{array}$ & 2017 & $\begin{array}{l}\text { Manis } \\
\text { javanica }\end{array}$ & \begin{tabular}{|l|} 
Beijing \\
Institute of \\
Microbiolo \\
gy and \\
Epidemiol \\
ogy \\
\end{tabular} & \begin{tabular}{|l|} 
Beijing \\
Institute of \\
Microbiolo \\
gy and \\
Epidemiol \\
ogy \\
\end{tabular} & $\begin{array}{l}\text { Wu-Chun Cao; } \\
\text { Tommy Tsan-Yuk } \\
\text { Lam; Na Jia; Ya-Wei } \\
\text { Zhang; Jia-Fu Jiang; } \\
\text { Bao-Gui Jiang }\end{array}$ \\
\hline $\begin{array}{l}\text { EPI_ISL_4107 } \\
21\end{array}$ & $\begin{array}{l}\text { hCoV- } \\
\text { 19/pangolin/Guangdong/ } \\
\text { 1/2019 }\end{array}$ & \begin{tabular}{|l|} 
Asia / \\
China / \\
Guangdo \\
ng
\end{tabular} & 2019 & $\begin{array}{l}\text { Manis } \\
\text { javanica }\end{array}$ & \begin{tabular}{|l|} 
South \\
China \\
Agricultura \\
I \\
University
\end{tabular} & \begin{tabular}{|l|} 
South \\
China \\
Agricultura \\
I \\
University
\end{tabular} & $\begin{array}{l}\text { Yongyi Shen, Lihua } \\
\text { Xiao, Wu Chen }\end{array}$ \\
\hline NC 019843.3 & $\begin{array}{l}\text { Middle East respiratory } \\
\text { syndrome-related } \\
\text { coronavirus }\end{array}$ & & & $\begin{array}{l}\text { Homo } \\
\text { sapiens }\end{array}$ & & & $\begin{array}{l}\text { van Boheemen,S., de } \\
\text { Graaf,M., Lauber,C., } \\
\text { Bestebroer,T.M., } \\
\text { Raj,V.S.,Zaki,A.M., } \\
\text { Osterhaus,A.D., } \\
\text { Haagmans,B.L., } \\
\text { Gorbalenya,A.E.,Snijd } \\
\text { er,E.J. and } \\
\text { Fouchier,R.A. }\end{array}$ \\
\hline NP_073551.1 & Human coronavirus $229 \mathrm{E}$ & & & $\begin{array}{l}\text { Homo } \\
\text { sapiens }\end{array}$ & & & $\begin{array}{l}\text { Thiel,V., Herold,J. and } \\
\text { Siddell,S.G. }\end{array}$ \\
\hline
\end{tabular}




\begin{tabular}{|c|c|c|c|}
\hline $\begin{array}{l}\text { YP_00955524 } \\
1.1\end{array}$ & Human coronavirus OC43 & $\begin{array}{l}\text { Homo } \\
\text { sapiens }\end{array}$ & $\begin{array}{l}\text { St-Jean,J.R., } \\
\text { Jacomy,H., } \\
\text { Desforges,M., } \\
\text { Vabret,A., } \\
\text { Freymuth,F. And } \\
\text { Talbot,P.J. }\end{array}$ \\
\hline AVP78042.1 & Bat SARS-like coronavirus & $\begin{array}{l}\text { Rhinolophus } \\
\text { pusillus }\end{array}$ & $\begin{array}{l}\text { Hu,D., Zhu,C., Ai,L., } \\
\text { He,T., Wang,Y., Ye,F., } \\
\text { Yang,L., } \\
\text { Ding,C.,Zhu,X., Lv,R., } \\
\text { Zhu,J., Hassan,B., } \\
\text { Feng,Y., Tan,W. and } \\
\text { Wang,C. }\end{array}$ \\
\hline APO40579.1 & $\begin{array}{l}\text { Severe acute respiratory } \\
\text { syndrome-related } \\
\text { coronavirus }\end{array}$ & $\begin{array}{l}\text { Rhinolophus } \\
\text { sp. }\end{array}$ & Tao,Y. and Tong,S. \\
\hline $\begin{array}{l}\text { YP_00385858 } \\
4.1\end{array}$ & $\begin{array}{l}\text { Bat coronavirus BM48- } \\
31 / B G R / 2008\end{array}$ & $\begin{array}{l}\text { Rhinolophus } \\
\text { blasii }\end{array}$ & $\begin{array}{l}\text { Drexler,J.F., } \\
\text { Corman,V.M. and } \\
\text { Drosten,C. }\end{array}$ \\
\hline AHX37558.1 & $\begin{array}{l}\text { Rhinolophus affinis } \\
\text { Coronavirus }\end{array}$ & $\begin{array}{l}\text { Rhinolophus } \\
\text { affinis }\end{array}$ & $\begin{array}{l}\text { He,B., Zhang,Y., Xu,L., } \\
\text { Yang,W., Yang,F., } \\
\text { Feng,Y., Xia,L., } \\
\text { Zhou,J., Zhen,W., } \\
\text { Feng,Y., Guo,H., } \\
\text { Zhang,H. and Tu,C. }\end{array}$ \\
\hline AAZ67052.1 & Bat Coronavirus RaTG13 & Bat & $\begin{array}{l}\text { Li,W., Shi,Z., Yu,M., } \\
\text { Ren,W., Smith,C., } \\
\text { Epstein,J.H., } \\
\text { Wang,H.,Crameri,G., } \\
\text { Hu,Z., Zhang,H., } \\
\text { Zhang,J., } \\
\text { McEachern,J., } \\
\text { Field,H.,Daszak,P., } \\
\text { Eaton,B.T., Zhang,S. } \\
\text { and Wang,L.F. }\end{array}$ \\
\hline ABD75332.1 & Bat SARS CoV Rm1/2004 & $\begin{array}{l}\text { Rhinolophus } \\
\text { macrotis }\end{array}$ & $\begin{array}{l}\text { Li,W., Shi,Z., Yu,M., } \\
\text { Ren,W., Smith,C., } \\
\text { Epstein,J.H., Wang,H., } \\
\text { Crameri,G., Hu,Z., } \\
\text { Zhang,H., Zhang,J., } \\
\text { McEachern,J., } \\
\text { Field,H., Daszak,P., } \\
\text { Eaton,B.T., Zhang,S. } \\
\text { and Wang,L.F. }\end{array}$ \\
\hline $\begin{array}{l}\text { YP_00982505 } \\
1.1\end{array}$ & SARS Coronavirus & $\begin{array}{l}\text { Homo } \\
\text { sapiens }\end{array}$ & $\begin{array}{l}\text { He,R., Dobie,F., } \\
\text { Ballantine,M., }\end{array}$ \\
\hline
\end{tabular}




\begin{tabular}{|c|c|c|c|}
\hline & & & $\begin{array}{l}\text { Leeson,A., Li,Y., } \\
\text { Bastien,N., Cutts,T., } \\
\text { Andonov,A., Cao,J., } \\
\text { Booth,T.F., } \\
\text { Plummer,F.A., } \\
\text { Tyler,S., Baker,L. and } \\
\text { Li,X. }\end{array}$ \\
\hline ATO98108.1 & Bat SARS-like Coronavirus & $\begin{array}{l}\text { Aselliscus } \\
\text { stoliczkanu }\end{array}$ & $\begin{array}{l}\text { Hu,B., Zeng,L.P., } \\
\text { Yang,X.L., Ge,X.Y., } \\
\text { Zhang,W., Li,B., } \\
\text { Xie,J.Z., Shen,X.R., } \\
\text { Zhang,Y.Z., Wang,N., } \\
\text { Luo,D.S., Zheng,X.S., } \\
\text { Wang,M.N., } \\
\text { Daszak,P., Wang,L.F., } \\
\text { Cui,J. and Shi,Z.L. }\end{array}$ \\
\hline ATO98145.1 & Bat SARS-like Coronavirus & $\begin{array}{l}\text { Rhinolophus } \\
\text { ferrumequin } \\
\text { um }\end{array}$ & $\begin{array}{l}\text { Hu,B., Zeng,L.P., } \\
\text { Yang,X.L., Ge,X.Y., } \\
\text { Zhang,W., Li,B., } \\
\text { Xie,J.Z., Shen,X.R., } \\
\text { Zhang,Y.Z., Wang,N., } \\
\text { Luo,D.S., Zheng,X.S., } \\
\text { Wang,M.N., } \\
\text { Daszak,P., Wang,L.F., } \\
\text { Cui,J. and Shi,Z.L. }\end{array}$ \\
\hline ATO98157.1 & Bat SARS-like Coronavirus & $\begin{array}{l}\text { Rhinolophus } \\
\text { sinicus }\end{array}$ & $\begin{array}{l}\text { Hu,B., Zeng,L.P., } \\
\text { Yang,X.L., Ge,X.Y., } \\
\text { Zhang,W., Li,B., } \\
\text { Xie,J.Z., Shen,X.R., } \\
\text { Zhang,Y.Z., Wang,N., } \\
\text { Luo,D.S., Zheng,X.S., } \\
\text { Wang,M.N., } \\
\text { Daszak,P., Wang,L.F., } \\
\text { Cui,J. and Shi,Z.L. }\end{array}$ \\
\hline AAY88866.1 & $\begin{array}{l}\text { Bat SARS Coronavirus } \\
\text { HKU3-1 }\end{array}$ & Bat & $\begin{array}{l}\text { Lau,S.K., Woo,P.C., } \\
\text { Li,K.S., Huang,Y., } \\
\text { Tsoi,H.W., Wong,B.H., } \\
\text { Wong,S.S., Leung,S.Y., } \\
\text { Chan,K.H. and } \\
\text { Yuen,K.Y. }\end{array}$ \\
\hline AGC74165.1 & $\begin{array}{l}\text { Bat Coronavirus } \\
\text { Rp/Shaanxi2011 }\end{array}$ & $\begin{array}{l}\text { Rhinolophus } \\
\text { pusillus }\end{array}$ & $\begin{array}{l}\text { Yang,L., Wu,Z., Ren,X., } \\
\text { Yang,F., He,G., } \\
\text { Zhang,J., Dong,J., } \\
\text { Sun,L., Zhu,Y., Du,J., } \\
\text { Zhang,S. and Jin,Q. }\end{array}$ \\
\hline
\end{tabular}




\begin{tabular}{|c|c|c|c|}
\hline AGC74176.1 & $\begin{array}{l}\text { Bat Coronavirus } \\
\text { Cp/Yunnan2011 }\end{array}$ & $\begin{array}{l}\text { Chaerephon } \\
\text { plicata }\end{array}$ & $\begin{array}{l}\text { Yang,L., Wu,Z., Ren,X., } \\
\text { Yang,F., He,G., } \\
\text { Zhang,J., Dong,J., } \\
\text { Sun,L., Zhu,Y., Du,J., } \\
\text { Zhang,S. and Jin,Q. }\end{array}$ \\
\hline AID16716.1 & Bat SARS-like coronavirus & $\begin{array}{l}\text { Rhinolophus } \\
\text { monoceros }\end{array}$ & $\begin{array}{l}\text { Lin,X.D., Wang,W., } \\
\text { Hao,Z.Y., Wang,Z.X., } \\
\text { Guo,W.P., Guan,X.Q., } \\
\text { Wang,M.R., } \\
\text { Wang,H.W., } \\
\text { Zhou,R.H., Li,M.H., } \\
\text { Tang,G.P., Wu,J., } \\
\text { Holmes,E.C. and } \\
\text { Zhang,Y.Z. }\end{array}$ \\
\hline AlA62277.1 & BtRf-BetaCoV/JL2012 & \begin{tabular}{|l|} 
Rhinolophus \\
ferrumequin \\
um
\end{tabular} & $\begin{array}{l}\text { Wu,Z., Yang,L., Ren,X., } \\
\text { He,G., Zhang,J., } \\
\text { Yang,J., Qian,Z., } \\
\text { Dong,J., Sun,L., Zhu,Y., } \\
\text { Du,J., Yang,F., } \\
\text { Zhang,S. and Jin,Q. }\end{array}$ \\
\hline AIA62310.1 & BtRs-BetaCoV/HuB2013 & $\begin{array}{l}\text { Rhinolophus } \\
\text { sinicus }\end{array}$ & $\begin{array}{l}\text { Wu,Z., Yang,L., Ren,X., } \\
\text { He,G., Zhang,J., } \\
\text { Yang,J., Qian,Z., } \\
\text { Dong,J., Sun,L., Zhu,Y., } \\
\text { Du,J., Yang,F., } \\
\text { Zhang,S. and Jin,Q. }\end{array}$ \\
\hline QHR63300.2 & Bat Coronavirus RaTG13 & $\begin{array}{l}\text { Rhinolophus } \\
\text { affinis }\end{array}$ & $\begin{array}{l}\text { Zhou,P., Yang,X.-L., } \\
\text { Wang,X.-G., Hu,B., } \\
\text { Zhang,L., } \\
\text { Zhang,W.,Si,H.-R., } \\
\text { Zhu,Y., Li,B., } \\
\text { Huang,C.-L., Chen,H.- } \\
\text { D., Chen,J., Luo,Y., } \\
\text { Guo,H., Jiang,R.-D., } \\
\text { Liu,M.-Q., Chen,Y., } \\
\text { Shen,X.-R., Wang,X., } \\
\text { Zheng,X.-S., Zhao,K., } \\
\text { Chen,Q.-J., Deng,F., } \\
\text { Liu,L.-L., Yan,B., } \\
\text { Zhan,F.-X., Wang,Y.- } \\
\text { Y., Xiao,G.-F. and } \\
\text { Shi,Z.-L. }\end{array}$ \\
\hline AVP78031.1 & Bat SARS-like Coronavirus & $\begin{array}{l}\text { Rhinolophus } \\
\text { pusillus }\end{array}$ & $\begin{array}{l}\text { Hu,D., Zhu,C., Ai,L., } \\
\text { He,T., Wang,Y., Ye,F., } \\
\text { Yang,L., Ding,C., } \\
\text { Zhu,X., Lv,R., Zhu,J., }\end{array}$ \\
\hline
\end{tabular}




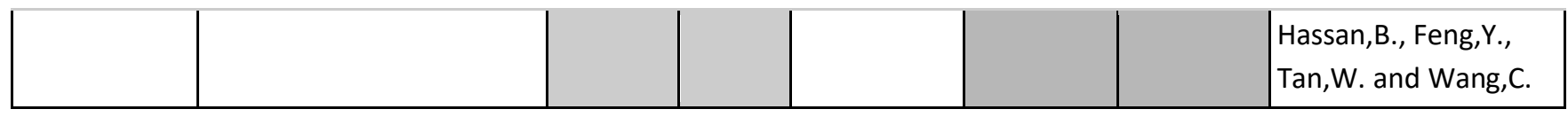

Table S6. Calculated sequence conservation percentages, across 28 coronavirus sequences, for the RBD and CTD1.

\begin{tabular}{|c|c|c|}
\hline position & $\%$ conserved & sequence (SARS-CoV-2 listed first) \\
\hline 324 & 7. & EI I I I IEGVAQTTKQQGQVKQQTQVQDQ \\
\hline 325 & 43. & SSSSSSSSSDSEEEEEDEDEEEEEDESS \\
\hline 326 & 36. & IIIIII IVIVIVVVVVVVVVVVVVVVIV \\
\hline 327 & 79. & VVVVVVVVVYVVVVIVVVVVIVI IAVVV \\
\hline 328 & 93. & RRRRRRRESRRRRRRRRRRRRRRRRRRR \\
\hline 329 & 89. & FFFFFFFQLRFFFFFFFFFFFFFFFFF \\
\hline 330 & 93. & PPPPPPPAPKPPPPPPPPPPPPPPPPPP \\
\hline 331 & 89. & NNNNNNNE-PNNNNNNNNNNNNNNNNNN \\
\hline 332 & 89. & IIIIIIG-LIIIIIIIIIIIIIIIII \\
\hline 333 & 89. & TTTTTTV-PTTTTTTTTTTTTTTT \\
\hline 334 & 89. & NNNNNNNE-NNNQNNNNNNNNNNNNNNN \\
\hline 335 & 50. & LLLLLLL---VLLLRRLRLLRRRRVRLV \\
\hline 336 & 96. & 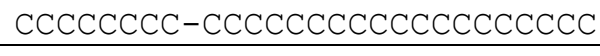 \\
\hline 337 & 89. & PPPPPPPD-NPPPPPPPPPPPPPPPPPP \\
\hline 338 & 93. & FFFFFFFF-IFFFFFFFFFFFFFFFFFF \\
\hline 339 & 43. & GGGGGGGS-EHGNGDDGDDGDDDDDDGH \\
\hline 340 & 43. & EEEEEEEP-AKQEEKKEKAEKKRKKREK \\
\hline 341 & 93. & VVVVVVVLVWVVVVVVVVVVVVVVVVVV \\
\hline 342 & 89. & FFFFFFFLYLFFFFFFFFFFFFFFFFFF \\
\hline 343 & 93. & NNNNNNNS HNNNNNNNNNNNNNNNNNNN \\
\hline 344 & 82 . & AAAAAAAGKDAAIAAAAAAAAAAVAAAA \\
\hline 345 & 61. & TSSSSSTTHKTSTTTSTTTTTTSTTSTT \\
\hline 346 & 46. & RKKKKKT-TSRNSTRRKRRTRRRRRRTR \\
\hline 347 & 93. & FFFFFFFPFFFFFFFFFFFFFFFFFE \\
\hline 348 & 29. & AАAАAАAP I P P PР P P P P P P P P P PAP \\
\hline 349 & 79. & SSSSSSSQVSSSSSNNSSSSNSSNSSSS \\
\hline 350 & 93. & VVVVVVVVLPVVVVVVVVVVVVVVVVVV \\
\hline 351 & 96. & YYYYYYYYYLYYYYYYYYYYYYYYYYYY \\
\hline 352 & 89. & AAAAAAAN-NAAAAAAAAAAAAAAAAAA \\
\hline 353 & 93. & WWWWWWWF-WWWWWWWWWWWWWWWWWWW \\
\hline 354 & 29. & NNNNNNNK-EEEEEEEEEEEEEEEEENE \\
\hline 355 & 96. & RRRRRRRR-RRRRRRRRRRRRRRRRRRR \\
\hline 356 & 43. & KKKKKKKL-KTLMKTTKTVKTTTTTTKT \\
\hline 357 & 43. & RRRRRRRV-TKRRRKKKKKRKKKKKKRK \\
\hline 358 & 89. & IIIIIIF-FIIIIIIIIIIIIIIII \\
\hline
\end{tabular}




\begin{tabular}{|c|c|c|}
\hline 359 & 89. & SSSSSSST-SSSTSSSSSSSSSSSSSSS \\
\hline 360 & 54. & NNNNNNNN-NDDNNDDNDNNDDDDDDND \\
\hline 361 & 96. & $\mathrm{CCCCCCCC}-\mathrm{CCCCCCCCCCCCCCCCCCC}$ \\
\hline 362 & 82. & VVVVVVVN-NIVVVVVVVVVVVVVVVVI \\
\hline 363 & 89. & AAAAAAAYVFAAAAAAAAAAAAAAAAAA \\
\hline 364 & 93. & DDDDDDDNDNDDDDDDDDDDDDDDDDDD \\
\hline 365 & 89. & YYYYYYYLFMYYYYYYYYYYYYYYYYYY \\
\hline 366 & 46. & SSSSSSSTKSTASSTTSTTSTTTTTTST \\
\hline 367 & 86. & VVVVVVVKPSVVVVVVVVAVVVVVVVVV \\
\hline 368 & 82. & LLLLLLLLQLFLLLLLLLFLLLLLFLLF \\
\hline 369 & 89. & YYYYYYYLSMYYYYYYYYYYYYYYYYYY \\
\hline 370 & 89. & NNNNNNNSGSNNNNNNNNNNNNNNNNNN \\
\hline 371 & 89. & SSSSSSSLGFSSSSSSSSSSSSSSSSSS \\
\hline 372 & 7. & ATTTTTTFGITSATTTTTTTTTTTTTTT \\
\hline 373 & 89. & SSSSSSSS-QSSSSSSFSSSSSSSSSSS \\
\hline 374 & 89. & FFFFFFFV-AFFFFFFFFFFFFFFFFFF \\
\hline 375 & 89. & SSSSSSSN-DSSSSSSSSSSSSSSSSSS \\
\hline 376 & 89. & TTTTTTTD-STTTTTTTTTTTTTTTTTT \\
\hline 377 & 96. & FFFFFFF-FFFFFFFFFFFFFFFFF \\
\hline 378 & 86. & KKKKKKKKTKTKKQKKKKKKKKKKKKKNKKK \\
\hline 379 & 100. & $\mathrm{CCCCCCCCCCCCCCCCCCCCCCCCCCCC}$ \\
\hline 380 & 89. & YYYYYYYSFNYYYYYYYYYYYYYYYYYY \\
\hline 381 & 89. & GGGGGGGQNNGGGGGGGGGGGGGGGGGG \\
\hline 382 & 89. & VVVVVVVICIVVVVVVVVVVVVVVVVVV \\
\hline 383 & 93. & SSSSSSSSYDSSSSSSSSSSSSSSSSSS \\
\hline 384 & 86. & PPPPPPPPPAPPPAPPAPPAPPPPPPPP \\
\hline 385 & 43. & TTTTTTTAAASTTISSTSSTSSSSSSTS \\
\hline 386 & 93. & KKKKKKKKAGKKKKKKKKKKKKKKKKKKKKKK \\
\hline 387 & 89. & LLLLLLLI-ILLLLLLLLLLLLLLLLLL \\
\hline 388 & 46. & NNNNNNNAVY INNNI INI INI I I II INI \\
\hline 389 & 89. & DDDDDDDSNGDDDDDDDDDDDDDDDDDD \\
\hline 390 & 89. & LLLLLLLNIMLLLLLLLLLLLLLLLLLL \\
\hline 391 & 96. & СССССCCCTCCCCCCCCCCCCCCCCCCC \\
\hline 392 & 93. & FFFFFFFYLFFFFFFFFFFFFFFFFFFF \\
\hline 393 & 71. & TTTTTTTSASTSSSTTSTTSTTTTTTTT \\
\hline 394 & 43. & NNNNNNNSNSSSSNSSSNSSNSSSSSSSNS \\
\hline 395 & 89. & VVVVVVVL-IVVVVVVVVVVVVVVVVVV \\
\hline 396 & 89. & YYYYYYYIFTYYYYYYYYYYYYYYYYYY \\
\hline 397 & 89. & AAAAAAALN IAAAAAAAAAAAAAAAAAA \\
\hline 398 & 96. & DDDDDDDDEDDDDDDDDDDDDDDDDDDD \\
\hline 399 & 39. & SSSSSSSYTKTYYSTTSTTSTTTTTTST \\
\hline 400 & 96. & FFFFFFF-FFFFFFFFFFFFFFFFF \\
\hline
\end{tabular}




\begin{tabular}{|c|c|c|}
\hline 401 & 46. & VVVVVVVS-ALVVVLLVLLVLLLLLLVL \\
\hline 402 & 54. & IVVVVVVY-IIVVVIIVIIVIIIIIIII \\
\hline 403 & 50. & RKKKKKRPKPRKKKRRKRRKRRRRRRTR \\
\hline 404 & 50. & GGGGGGGLGNFGGGSSGSFGSSFSFSGF \\
\hline 405 & 46. & DDDDDDDSPGSDDDSSDSSDSSSSSSDS \\
\hline 406 & 71. & EEEEEEEMLREDDDEEDEEDEEEEEEEE \\
\hline 407 & 89. & VVVVVVVKCKVVVVVVVVVVVVVVVVVV \\
\hline 408 & 89. & RRRRRRRSVVRRRRRRRRRRRRRRRRRR \\
\hline 409 & 89. & 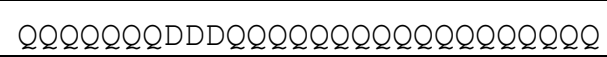 \\
\hline 410 & 50 & IIIIIIILTLVIIIVVIVVIVVIVVVIV \\
\hline 411 & 89. & AAAAAAAS SQAAAAAAAAAAAAAAAAAA \\
\hline 412 & 89. & PPPPPPPVHLPPPPPPPPPPPPPPPPPP \\
\hline 413 & 86. & GGGGGGGSFGGAAGGGGGGGGGGGGGGG \\
\hline 414 & 57. & QQQQQQQSTNQQQQEEQEEQEEEEQEQQ \\
\hline 415 & 93. & TTTTTTTAтLTTTTTTTTTTTTTTTT \\
\hline 416 & 96. & GGGGGGGGKGGGGGGGGGGGGGGGGGGG \\
\hline 417 & 7. & KVVVVVRPYYVVVVVVVVVVVVVVVVKV \\
\hline 418 & 93. & IIIIIIIVLIIIIIIIIIIIIIIII \\
\hline 419 & 93. & AAAAAAASAQAAAAAAAAAAAAAAAAAA \\
\hline 420 & 89. & DDDDDDDQVSDDDDDDDDDDDDDDDDDD \\
\hline 421 & 93. & YYYYYYYFYFYYYYYYYYYYYYYYYYYY \\
\hline 422 & 96. & NNNNNNNN-NNNNNNNNNNNNNNNNNNNN \\
\hline 423 & 96. & YYYYYYYY-YYYYYYYYYYYYYYYYYYY \\
\hline 424 & 93. & KKKKKKKKK-RKKKKKKKKKKKKKKKKKKKK \\
\hline 425 & 89. & LLLLLLLQ-ILLLLLLLLLLLLLLLLLL \\
\hline 426 & 89. & PPPPPPPS-DPPPPPPPPPPPPPPPPPP \\
\hline 427 & 89. & DDDDDDDF-TDDDDDDDDDDDDDDDDDD \\
\hline 428 & 86. & DDDDDDDS-TDDDDDDDDDDDDEDDDDD \\
\hline 429 & 89. & FFFFFFFN-AFFFFFFFFFFEFFFFFFF \\
\hline 430 & 79. & TTTTTTTP-TTTTMTTMTTLTTTTITTT \\
\hline 431 & 89. & GGGGGGGTASGGGGGGGGGGGGGGGGGG \\
\hline 432 & 96. & $\mathrm{CCCCCCCCNCCCCCCCCCCCCCCCCCCC}$ \\
\hline 433 & 93. & VVVVVVVLVQVVVVVVVVVVVVVVVVVV \\
\hline 434 & 79. & IIIIIIIGLILILIILIILIIIIIIII \\
\hline 435 & 89. & AAAAAAALRYAAAAAAAAAAAAAAAAAA \\
\hline 436 & 93. & WWWWWWWAWYWWWWWWWWWWWWWWWWWW \\
\hline 437 & 93. & NNNNNNN--NNNNNNNNNNNNNNNNNNN \\
\hline 438 & 29. & SSSSSSSV--TTTTTTTTTTTTTTTTST \\
\hline 439 & 18. & NVVVVVNPAPANNRAARAANAAAAAAKA \\
\hline 440 & 21. & NKKKKKNHSAKS SNKQNQKSKNNKKKHK \\
\hline 441 & 11. & LQQQQQLNIAQVLIQQ IQQKHQQQQQQIQ \\
\hline 442 & 89. & DDDDDDDLNNDDDDDDDDDDDDDDDDDD \\
\hline
\end{tabular}




\begin{tabular}{|c|c|c|}
\hline 443 & 18. & SAAAAASTTVTSSAQQAKVSTQRIVTAV \\
\hline 444 & 14. & KLLLLLKTGSGK-TGGTGGSGGGGGGKG \\
\hline 445 & 11. & VTTTTTVI-V-S-S--S--T------E- \\
\hline 446 & 29. & GGGGGGGT-S--- S--T--S------ G- \\
\hline 447 & 36. & GGDGGGG--S----G--G--G-------G- \\
\hline 448 & 43. & 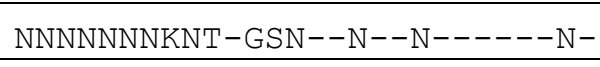 \\
\hline 449 & 32. & YYYYYYYPCW-NNF--Y--Y------E- \\
\hline 450 & 39. & N-GGGGNKPNHNENQQNQSNNQQNSYNN \\
\hline 451 & 82. & Y-YYYYYYFKYEFYYYYYYYYYYYYYYY \\
\hline 452 & 32. & LLLLLLLSSRFYFKYYKYFLYYYYFYLF \\
\hline 453 & 93. & YYYYYYYYFFYYYYYYYYYYYYYYYYYY \\
\hline 454 & 89. & RRRRRRRI-GRRRRRRRRRRRRRRRRRR \\
\hline 455 & 32. & LLLLLLLN-FSLRSSSYSSWSSSSSSLS \\
\hline 456 & 36. & FFFFFFFK-IHFFLHYLSHVHSSHHHFH \\
\hline 457 & 89. & RRRRRRRC-ERRRRRRRRRRRRRRRRRR \\
\hline 458 & 57. & KKKKKKKS-DSHHHKKHKSRKKKKSKKS \\
\hline 459 & 39. & SSSSSSSRGSTGGGTEGTSSTETTSTAT \\
\hline 460 & 11. & NKKKKKNLKVKKKKKKKKKKKKKKKKKKKNK \\
\hline 461 & 86. & LLLLLLLLVFL I ILLLLLLLLLLLLLLL \\
\hline 462 & 82. & KKKKKKK--KKKKRKKRKKNKKKKKKKKK \\
\hline 463 & 89. & PPPPPPPDNNPPPPPPPPPPPPPPPPPP \\
\hline 464 & 82. & FFFFFFFDF-FYYFFFFFFYFFFFFFFF \\
\hline 465 & 86. & EEEEEEERVHEEGEEEEEEEEEEEEEEE \\
\hline 466 & 89. & RRRRRRRTKDRRRRRRRRRRRRRRRRRR \\
\hline 467 & 89. & DDDDDDD--VDDDDDDDDDDDDDDDDDD \\
\hline 468 & 39. & IIIIIIIVFYLILILLILLLLLLLLLIL \\
\hline 469 & 89. & SSSSSSSPGASSSSSSSSSSSSSSSSSS \\
\hline 470 & 29. & TTTTTTTQSQSNNNSSNSSNSSSSSSTS \\
\hline 471 & 32. & EEEEEEELVHDVVVDDVDDDDDDDEDED \\
\hline 472 & 32. & IIIIIIIVCCELLPEEPEEIDEEDEDIE \\
\hline 473 & 36. & YYYYYYYNFF-YFF--F--YG--G-GY- \\
\hline 474 & 29. & $Q Q Q Q Q Q Q \mathrm{ASK}-\mathrm{NNS}--\mathrm{S}--\mathrm{S}------\mathrm{Q}-$ \\
\hline 475 & 32. & AAAAAAANLA $-\mathrm{SPP}--\mathrm{P}--\mathrm{P}------\mathrm{A}-$ \\
\hline 476 & 32. & GGGGGGGQKP-ASD--D--G-------G- \\
\hline 477 & 29. & SSSSSSSYIK-GGG--G--G------S- \\
\hline 478 & 25. & TTTTTTTS-N-GGK--K--Q------K- \\
\hline 479 & 39. & PPPPPPPP-F-TTP--P--S------P- \\
\hline 480 & 54. & $\mathrm{CCCCCCCC}-\mathrm{CNCCC}--\mathrm{C}--\mathrm{C}------\mathrm{CN}$ \\
\hline 481 & 29. & NNNNNNNV $-\mathrm{P}-\mathrm{S} S---------------\mathrm{N}-$ \\
\hline 482 & 36. & GGGGGGGS-CGS-T--T--S------ GG \\
\hline 483 & 14. & $\mathrm{VQQQQQQV}---\mathrm{VIAP}--\mathrm{P}--\mathrm{A}------\mathrm{QV}$ \\
\hline 484 & 11. & EVVVVVEI PKRSEP--P--I------TR \\
\hline
\end{tabular}




\begin{tabular}{|c|c|c|}
\hline 485 & 39. & GGGGGGGVGL-QGA--A-- G-------G- \\
\hline 486 & 11. & FLLLLLFPGN-LLF--L--P------L- \\
\hline 487 & 79. & NNNNNNNSCG-GNNNNNNNNNNNNNNN- \\
\hline 488 & 46. & CCCCCCCTA--CCCGGCGGCGGGGGGC- \\
\hline 489 & 46. & YYYYYYYVM--YYYVVYVVYVVVVVVY- \\
\hline 490 & 7. & FYYYYYFWP--EKWRYWRRNYYRYLYY- \\
\hline 491 & 46. & PPPPPPPEISTPPPTTPTTPTTTTTTPT \\
\hline 492 & 89. & LLLLLLLDVCLLLLLLLLLLLLLLLLLL \\
\hline 493 & 7. & QEEEEEQGAVSKANSSNSSRSSSSSSYS \\
\hline 494 & 14. & SRRRRRSDNGTSSDTTDTTPTTTTTTRT \\
\hline 495 & 93. & YYYYYYYYWSYYYYYYYYYYYYYYYYYY \\
\hline 496 & 50. & GGGGGGGQAGDGGGDDGDDGDDDDDDGD \\
\hline 497 & 89. & FFFFFFFWYPFFFFFFFFFFFFFFFFF \\
\hline 498 & 4. & QHHHHHHLSGNTTYYYYYNFNYYNNNYN \\
\hline 499 & 71. & PPPPPPPV-KPPQTPPTPPTPPPPQPPP \\
\hline 500 & 46. & TTTTTTTA-NNTSTSSTTNTNSSNNNTN \\
\hline 501 & 14. & NTTTTTNS-NVVSNVITVVAVVVVVVDV \\
\hline 502 & 54. & GGGGGGGG-GPGGGPPGPPGPPPPPPGP \\
\hline 503 & 54. & VVVVVVVS-ILVI IVVI I LVVLLVLVVL \\
\hline 504 & 32. & GNNNNNGTKGEGGGAEGEDGADEAEAGE \\
\hline 505 & 82. & YYYYYYYVYTYYFYYYYYYHYYYYYYHY \\
\hline 506 & 89. & QQQQQQQQAYCQQQQQQQQQQQQQQQQQQQQQQ \\
\hline 507 & 50. & PPPPPP PMT PAPPPAAPAAPAAAAAAPA \\
\hline 508 & 29. & YFFFFFYTIATYYYTTYTTYTTTTTTYT \\
\hline 509 & 89. & RRRRRRREGGRRRRRRRRRRRRRRRRRR \\
\hline 510 & 89. & VVVVVVVQSTVVVVVVVVVVVVVVVVVV \\
\hline 511 & 89. & VVVVVVVLLNVVVVVVVVVVVVVVVVVV \\
\hline 512 & 89. & VVVVVVVQYYVVVVVVVVVVVVVVVVVV \\
\hline 513 & 93. & LLLLLLLMVLLLLLLLLLLLLLLLLLLL \\
\hline 514 & 93. & SSSSSSSGSTSSSSSSSSSSSSSSSSSS \\
\hline 515 & 86. & FFFFF-F--PFFFFFFFFFFFFFFFFFF \\
\hline 516 & 89. & EEEEEEE--QEEEEEEEEEEEEEEEEEE \\
\hline 517 & 89. & LLLLLLL--ALLLLLLLLLLLLLLLLLL \\
\hline 518 & 89. & LLLLLLL--FLLLLLLLLLLLLLLLLLL \\
\hline 519 & 4. & HNNNNNN--LNNNNNNNNNNNNNNNNNN \\
\hline 520 & 71. & AGGGGGA-- GAAAAAAAAAAAAAAAAAA \\
\hline 521 & 89. & PPPPPPP--WPPPPPPPPPPPPPPPPPP \\
\hline 522 & 89. & AAAAAAA--SAAAAAAAAAAAAAAAAAA \\
\hline 523 & 89. & TTTTTT--DTTTTTTTTTTTTTTTT \\
\hline 524 & 89. & VVVVVVV--SVVVVVVVVVVVVVVVVVV \\
\hline 525 & 93. & $\mathrm{CCCCCCC}--\operatorname{CcCCCCCCCCCCCCCCCCC}$ \\
\hline 526 & 93. & GGGGGGGDGLGGGGGGGGGGGGGGGGGG \\
\hline
\end{tabular}




\begin{tabular}{|c|c|c|}
\hline 527 & 89. & PPPPPPPTVQPPPPPPPPPPPPPPPPPP \\
\hline 528 & 96. & KKKKKKKKKSKKKKKKKKKKKKKKKKKKKKKK \\
\hline 529 & 11. & KLLLLLQISALKQLLLLLLLLLLLLLKL \\
\hline 530 & 89. & SSSSSSSAFNSSSSSSSSSSSSSSSSSS \\
\hline 531 & 93. & тTтTтTSмTTTтTтTтTтTтTтTтTт \\
\hline 532 & 14. & NTTTTTNQNDQEEDQQDGQDETSQPENQ \\
\hline 533 & 89. & LLLLLLL-VILLLLLLLLLLLLLLLLLL \\
\hline 534 & 75. & VVVVVVV-TIVVVIVVIVVIVVIVVVVV \\
\hline 535 & 86. & KKKKKKKKLLLKKKTKKKKKKKKKKKKKKKK \\
\hline 536 & 71. & NDDDDDNGDGNNNNNNNNNNNNNNNNNN \\
\hline 537 & 39. & KKKKKKKNKVQKKQQQQQQQQQQQQQQKQ \\
\hline 538 & 100. & $\mathrm{CCCCCCCCCCC} \mathrm{CCCCCCCCCCCCCCCC}$ \\
\hline 539 & 96. & VVVVVVVVTVVVVVVVVVVVVVVVVVVV \\
\hline 540 & 93. & NNNNNNNEKNNNNNNNNNNNNNNNNNNN \\
\hline 541 & 89. & FFFFFFFYYYFFFFFFFFFFFFFFFFF \\
\hline 542 & 93. & NNNNNNNSNDNNNNNNNNNNNNNNNNNN \\
\hline 543 & 89. & FFFFFFFLILFFFFFFFFFFFFFFFFF \\
\hline 544 & 89. & NNNNNNNYYYNNNNNNNNNNNNNNNNNN \\
\hline 545 & 96. & GGGGGGGGDGGGGGGGGGGGGGGGGGGG \\
\hline 546 & 89. & LLLLLLLVVILLLLLLLLLLLLLLLLLL \\
\hline 547 & 46. & TTTTTTTSSLKTTTKRTKKTKKKKKKTK \\
\hline 548 & 100. & GGGGGGGGGGGGGGGGGGGGGGGGGGGG \\
\hline 549 & 89. & TTTTTTTRVQTTTTTTTTTTTTTTTTTT \\
\hline 550 & 100 & GGGGGGGGGGGGGGGGGGGGGGGGGGGG \\
\hline 551 & 96. & VVVVVVVVVIVVVVVVVVVVVVVVVVVV \\
\hline 552 & 89. & LLLLLLLFIFLLLLLLLLLLLLLLLLLL \\
\hline 553 & 89. & TTTTTTTQRVTTTTTTTTTTTTTTTTTT \\
\hline 554 & 18. & ETTTTTENVEDSNPETPDDSSADSDKED \\
\hline 555 & 93. & SSSSSSSCSVSSSSSSSSSSSSSSSSSS \\
\hline 556 & 14. & NKKKKKSTNNSTTLSSSSSSSSSSSSNS \\
\hline 557 & 89. & KKKKKKKADAKKKKKKKKKKKKKKKKKKK \\
\hline 558 & 25. & KQQQQQKVTTRKKRRRRRRRRKKRTRKR \\
\hline 559 & 93. & FFFFFFFGYFFFFFFFFFFFFFFFF \\
\hline 560 & 32. & LLLLLLLVLYQQQQQQQQQQQQQQQQQQLQ \\
\hline 561 & 46. & PPPPPPPRNNSPPPSSPSSPSSSSSSPS \\
\hline 562 & 89. & FFFFFFF--SFFFFFFFFFFFFFFFFFF \\
\hline 563 & 96. & QQQQQQQQQ I QQQQQQQQQQQQQQQQQQQQQQQ \\
\hline 564 & 89. & QQQQQQQRTNQQQQQQQQQQQQQQQQQQQ \\
\hline 565 & 93. & FFFFFFFFYLFFFFFFFFFFFFFFFFFF \\
\hline 566 & 89. & GGGGGGGVTLGGGGGGGGGGGGGGGGGG \\
\hline 567 & 82. & RRRRRRRYSYKRRRRRRRRRRRRRRRRK \\
\hline 568 & 96. & DDDDDDDDTDDDDDDDDDDDDDDDDDDD \\
\hline
\end{tabular}




\begin{tabular}{|l|l|l|}
\hline 569 & 29. & IIIIIIIASSAVVFTTVTTVTAATATIA \\
\hline 570 & 11. & ASSSSSAYGNSSSSSSSSSSSSSSSSAS \\
\hline 571 & 89. & DDDDDDDQ--DDDDDDDDDDDDDDDDDD \\
\hline 572 & 29. & TTTTTTTLLNFFFFFFFFFFFFFFFFTF \\
\hline 573 & 82. & TTTTTTTVLLITTTTTTTTTTTTTTTTI \\
\hline 574 & 89. & DDDDDDD--YDDDDDDDDDDDDDDDDDD \\
\hline 575 & 29. & AAAAAAAGGGSSSSSSSSSSSSSSSSAS \\
\hline 576 & 89. & VVVVVVVYFFVVVVVVVVVVVVVVV \\
\hline 577 & 93. & RRRRRRRYKRRRRRRRRRRRRRRRRRRR \\
\hline 578 & 96. & DDDDDDDSDDDDDDDDDDDDDDDDDDDD \\
\hline 579 & 89. & PPPPPPPDVYPPPPPPPPPPPPPPPPPP \\
\hline 580 & 71. & QQQQQQQDTIQKKKQQKQQKQQQQQQQQ \\
\hline 581 & 93. & TTTTTTTNKTTTTTTTTTTTTTTTTTT \\
\hline 582 & 79. & LLLLLLL-GNLFLLLLSLLSLLLLLLLL \\
\hline 583 & 75. & EEEEEEE-TREEEEEEEQDEEEQEQEEE \\
\hline 584 & 86. & IIIIIIIYITIIIVIIIVIIIIIIIIII \\
\hline 585 & 89. & LLLLLLLYYFLLLLLLLLLLLLLLLLLL \\
\hline 586 & 89. & DDDDDDDCSMDDDDDDDDDDDDDDDDDD \\
\hline 587 & 96. & IIIIIIILIIIIIIIIIIIIIIIIIIII \\
\hline 588 & 46. & TTTTTTTRTRTSASSSSTTSSSSSSSTT \\
\hline 589 & 93. & PPPPPPPAPSPPPPPPPPPPPPPPPPPP \\
\hline 590 & 100. & CCCCCCCCCCCCCCCCCCCCCCCCCCCC \\
\hline 591 & 86. & SSSSSSSVNYSSSSSSASSSSSSSSSSS \\
\hline
\end{tabular}

\section{REFERENCES}

1. Le Guilloux, V.; Schmidtke, P.; Tuffery, P., Fpocket: An open source platform for ligand pocket detection. BMC Bioinformatics 2009, 10 (1), 168.

2. Yuan, M.; Wu, N. C.; Zhu, X.; Lee, C.-C. D.; So, R. T. Y.; Lv, H.; Mok, C. K. P.; Wilson, I. A., A highly conserved cryptic epitope in the receptor binding domains of SARS-CoV-2 and SARS-CoV. Science 2020, 368 (6491), 630-633.

3. $\quad$ Casalino, L.; Gaieb, Z.; Goldsmith, J. A.; Hjorth, C. K.; Dommer, A. C.; Harbison, A. M.; Fogarty, C. A.; Barros, E. P.; Taylor, B. C.; McLellan, J. S.; Fadda, E.; Amaro, R. E., Beyond Shielding: The Roles of Glycans in the SARS-CoV-2 Spike Protein. ACS Central Science 2020, 6 (10), 1722-1734.

4. Elbe, S.; Buckland-Merrett, G., Data, disease and diplomacy: GISAID's innovative contribution to global health. Global Challenges 2017, 1 (1), 33-46. 